\title{
Secondary Eyewall Formation and Concentric Eyewall Replacement in Association with Increased Low-Level Inner-Core Diabatic Cooling
}

\author{
GuANGHUA CHEN \\ Center for Monsoon System Research, Institute of Atmospheric Physics, Chinese Academy of Sciences, Beijing, and Key \\ Laboratory of Meteorological Disaster, Ministry of Education, Nanjing University of Information \\ Science and Technology, Nanjing, China
}

(Manuscript received 19 July 2017, in final form 26 March 2018)

\begin{abstract}
The role of increased diabatic cooling in secondary eyewall formation (SEF) and eyewall replacement cycle (ERC) is examined using idealized numerical simulation. The experiment with the low-level inner-core diabatic cooling increased by $30 \%$ features the low-entropy air and downward motion in the inner-core region whereas the convergence and active convective updrafts are in the outer-core region. In collaboration with the favorable ambient dynamical conditions and boundary layer dynamical processes, the concentric convective ring is initiated with the aid of the outward expansion of strong wind field, and then contracts inward to replace the inner eyewall. Subsequently, the deep-tropospheric radial outflows driven by the large outward-directed agradient force related to the massive strong tangential wind generate a largely outward-tilted eyewall, eventually forming a large-eyed storm. The sensitivity to the strength and radial location of diabatic cooling shows that neither the $20 \%$ increase nor $10-\mathrm{km}$ radially inward shift of the low-level cooling produces a pronounced SEF and ERC because of the lack of an evident moat region. In contrast, both the $40 \%$ increase and $10-\mathrm{km}$ radially outward shift of cooling lead to the active outer rainbands occurring at a larger radius. In the former case, because of the deep-layer radial outflow above the boundary layer, the largely outward-tilted concentric eyewall shrinks slowly, directly creating a large-eyed structure. In the latter case, the formation of concentric eyewall is delayed because of the low inertial stability at a large radius, but experiences an expeditious ontraction because of the strong radial inflow.
\end{abstract}

\section{Introduction}

The existence of a concentric eyewall structure is a universal episode in intense tropical cyclones (TCs). Based on the passive microwave and best-track data over the western North Pacific between 1997 and 2006, Kuo et al. (2009) identified that approximately $57 \%$ of category- 4 and $72 \%$ of category- 5 typhoons possessed concentric eyewalls during their lifetime. The commonly observed concentric eyewall replacement cycle (ERC) generally involves the following stages: secondary eyewall formation (SEF), inward contraction of the secondary eyewall, demise and replacement of the inner eyewall, and organization of a new substitute eyewall (e.g., Willoughby et al. 1982; Kossin and Sitkowski 2009; Zhu and Zhu 2014). The ERC process can render notable structure change and intensity fluctuation (e.g., Houze et al. 2007; Rozoff et al. 2008; Zhou et al. 2011;

Corresponding author: Dr. Guanghua Chen, cgh@mail.iap.ac.cn
Sitkowski et al. 2011; Wu et al. 2012), which poses a challenge for forecasters because of the lack of understanding of the complicated processes.

A variety of hypotheses on SEF has been proposed, including external forcing and internal dynamics. For example, topographic forcing can act as a potential role in SEF hypothesized by Hawkins (1983). Other studies also suggested that the interaction of TCs with other external forcings, such as synoptic-scale baroclinic eddies, local perturbations in sea surface temperature (SST), and upper-level jet (Molinari and Vollaro 1990; Nong and Emanuel 2003; Dai et al. 2017), is likely to facilitate SEF. Because the environment may vary dramatically from case to case, the frequent occurrence nature of a concentric eyewall implies that internal dynamics can play a fundamental role in SEF. One of the internal dynamics is propagating vortex Rossby waves (VRWs) proposed by Montgomery and Kallenbach (1997), who pointed out that the VRWs can interact with the mean flow near the stagnation radius, where the 
energy and angular momentum of VRWs tend to be concentrated, contributing to SEF. As an extension, Terwey and Montgomery (2008) proposed a dynamical theory called $\beta$ skirt axisymmetrization (BSA), in which the secondary wind maximum is hypothesized to be generated by an anisotropic upscale energy cascade and axisymmetrization of convectively generated PV anomalies. Once the low-level jet outside the primary eyewall becomes strong enough, a positive feedback, such as the wind-induced surface heat exchange (Emanuel 1986; Nong and Emanuel 2003), can take effect to amplify the perturbations into a concentric eyewall. The mechanisms related to the VRW stagnation radius and the BSA may work cooperatively in the sense that the former helps to establish an extensive $\beta$ skirt and the latter takes charge from then on (Qiu et al. 2010). Based on studies of vortex dynamics, the axisymmetrization process has been used to account for SEF. In the two-dimensional, nondivergent barotropic model, Kuo et al. $(2004,2008)$ demonstrated that a concentric vorticity ring can form through the axisymmetrization of weak vorticity patches around a primary vortex, provided that the primary vortex is strong enough and the two vortices are sufficiently close to each other.

Later, based on a realistic simulation, Huang et al. (2012) proposed a new paradigm for SEF in which an unbalanced boundary layer response to an expanding swirling wind field is emphasized. Accompanied by the outward expansion of tangential wind, the generation of supergradient winds in and just above the boundary layer can trigger a narrow region of strong boundary layer convergence caused by the deceleration of radial inflows. As a result, the unbalanced response in the boundary layer acts as a crucial role in initiating and sustaining a deep convective ring in a narrow supergradient wind zone outside the primary eyewall.

On the other hand, a great deal of research has examined the effects of the diabatic process in different radial regions on TC structure and intensity. TC circulation outside the primary eyewall is often categorized into outer and inner rainband regions. It is commonly accepted that outer spiral rainbands and associated downdrafts caused by evaporative cooling of raindrops and melting cooling of snow and graupel can be viewed as hostile factors to TC intensity. Thermodynamically, the lower-entropy air beneath the melting level in outer rainbands can be advected to the core region by boundary layer inflows and then entrained into the eyewall, thus suppressing eyewall convection and reducing TC intensity. Dynamically, downdrafts may act as a barrier effect on boundary layer inflow, which is detrimental to both mass and moisture convergence into the eyewall (e.g., Willoughby et al. 1982; Powell 1990; Bender 1997). By modifying diabatic heating in the outer spiral rainbands outside a radius of $90 \mathrm{~km}$, Wang (2009) pointed out that the reduction in the surface pressure on the inward side of the increased diabatic heating in the outer rainbands would decrease the horizontal pressure gradient across the RMW, thus weakening the storm intensity. In addition, the increased outer diabatic heating can develop a typical concentric eyewall structure and associated eyewall replacement as a result of the axisymmetrization of the inwardpropagating active spiral rainbands. On the contrary, the increase in diabatic cooling in the outer spiral rainbands tends to maintain both the intensity and innercore compactness of a storm. By comparison, the inner rainband region is generally dominated by strain flow between the radius of maximum wind (RMW) and about 2-3 times the RMW (Wang 2008a), which is alternatively referred to as the rapid filamentation zone (RFZ). Li et al. (2014) found that the removal of deeptropospheric heating (cooling) within the RFZ would reduce (increase) TC intensity, which is opposite to the effects of the diabatic process in the outer rainbands where heating (cooling) is generally negative (positive) to TC intensity as documented by Wang (2009). Besides, the diabatic heating in the RFZ plays a key role in broadening the inner-core size whereas the diabatic cooling limits the inner-core size.

Furthermore, regarding the role of evaporative cooling in SEF, Fang and Zhang (2012) suggested that the development of new convection preceding SEF might be facilitated with a front-like feature in the low-level equivalent potential temperature $\left(\theta_{e}\right)$ field. The sharpening of the $\theta_{e}$ gradient was due to the evaporative cooling of rainwater under a wavenumber-1 stratiform cloud deck. Besides, Qiu and Tan (2013) found, collocated with the strong evaporative cooling, that the strong radial inflow originating from the middle and downwind portions of the outer rainbands can descend slantwise to the surface and thus sharpen the gradient of radial velocity within the boundary layer, reinforcing the convergence to facilitate the SEF. Recently, by partitioning the precipitation into the convective and stratiform regimes, Chen et al. (2018) examined the effects of inner-core convective and stratiform diabatic processes on TC structure and intensity change. In the sensitivity experiment with the doubled diabatic cooling associated with the stratiform precipitation, they found that the secondary convective ring emerges within the inner-core region during the late period of integration, followed by the breakdown of the primary eyewall, analogous to SEF and ERC. Therein, the detailed dynamics of the SEF and ERC was not yet elucidated. As an extension of 
Chen et al. (2018), this study intends to examine the role of increased low-level inner-core diabatic cooing in subsequent SEF and ERC.

This study is organized as follows. The model setting and experimental design are described in section 2 . Section 3 presents a detailed description of TC intensity and structure evolution in the control and primary sensitivity experiments. The dynamical mechanisms of SEF and ERC are further investigated in section 4. Specific focus is placed on the environmental dynamic parameters, contribution of the outer rainbands, and boundary layer dynamical features. The sensitivity of SEF and ERC to the strength and radial location of low-level cooling is discussed in section 5. The conclusions and discussion are summarized in the last section.

\section{Model setting and experimental design}

The model used in this study is the Advanced Research Weather Research and Forecast (WRF) Model, version 3.6. The model is conducted in quadruple square domains with each child domain centered at the center of its parent domain. The domains contain identical grid points of $274 \times 274$ and have horizontal grid spacing of $54,18,6$, and $2 \mathrm{~km}$, respectively. The model has 30 vertical levels in the terrain-following sigma coordinate with the model top at $50 \mathrm{hPa}$. The innermost domain is designed to move with the model TC center such that the TC is always located at the center of the finest domain. The major model physics parameterizations include the WRF single-moment 6-class microphysics scheme (WSM6) with graupel (Hong et al. 2004), the Yonsei University (YSU) planetary boundary layer scheme (Noh et al. 2003), the Rapid Radiative Transfer Model radiation scheme (Mlawer et al. 1997), and the Dudhia scheme (Dudhia 1989) for longwave and shortwave radiation, respectively. Since the experiment contains no large-scale environmental flows, the outer rainbands are nearly confined within the finest domain. As such, no cumulus parameterization is employed.

The model is initialized with an axisymmetric cyclonic vortex on an $f$ plane centered at $18^{\circ} \mathrm{N}$ in a quiescent environment over the ocean with a fixed SST of $29^{\circ} \mathrm{C}$. An axisymmetric baroclinic vortex, similar to that used by Rotunno and Emanuel (1987), with the maximum wind of $12 \mathrm{~m} \mathrm{~s}^{-1}$ at a radius of $108 \mathrm{~km}$ is inserted at the center of domains. The mass and thermodynamic fields are obtained by solving a nonlinear balance equation for the given tangential wind fields. Following Chen (2016), the environmental thermodynamic profiles are specified as the average in the TC peak region $\left(5^{\circ}-20^{\circ} \mathrm{N}, 130^{\circ}-\right.$ $170^{\circ} \mathrm{E}$ ) during July-September from 1979 to 2012 based on the Japanese 55-year Reanalysis (Ebita et al. 2011).
After a spinup period of $62 \mathrm{~h}$, the simulated TC achieves a maximum near-surface azimuthal-mean tangential wind (MTW) of about $62 \mathrm{~m} \mathrm{~s}^{-1}$ and a minimum sea level pressure (MSLP) of $956 \mathrm{hPa}$. It is close to the average intensity of the observed TCs with a concentric eyewall structure (Kossin and Sitkowski 2009). As displayed in Figs. 1a,b, the simulated TC develops a structure similar to real TCs (e.g., Willoughby et al. 1984; Guinn and Schubert 1993), which features an echo-free elliptic eye surrounded by a closed eyewall with high reflectivity and coherent convective precipitation between $20-$ and $40-\mathrm{km}$ radii. Connecting to the outer edge of the eyewall, the well-organized inner rainbands spiral outward within radii of 40 and $80 \mathrm{~km}$, where the radially narrow and azimuthally elongated convective precipitation related to the inner spiral rainbands is embedded with the stratiform precipitation region. Farther radially outward, the outer spiral rainbands outside $80 \mathrm{~km}$ are more loosely organized, characterized by the isolated and scattered convective precipitation patches in the upwind and middle sectors of the outer rainbands with the stratiform precipitation being predominant in the northeastern downwind region, well consistent with the findings in previous studies (e.g., Hence and Houze 2008; Moon and Nolan 2010). In terms of the azimuthal-mean structure, the maximum ascending motion in the primary eyewall is located at a radius of $20 \mathrm{~km}$ and collocated with the MTW, with a radially outward slope with height. The secondary maximum upward motion can be seen outside $80 \mathrm{~km}$ in the mid- to upper troposphere, corresponding to the active outer rainbands (Fig. 1c). The composite vertical profiles of vertical velocity and diabatic heating within 40 and $80 \mathrm{~km}$ illustrates that, in the convective precipitation, the upward motion and diabatic heating dominate throughout the troposphere with the maximum vertical velocity near $5-\mathrm{km}$ height and the maximum heating around 11- $\mathrm{km}$ height, while the counterparts in the stratiform precipitation display a dipole vertical structure with the upward motion and diabatic heating at the upper levels and the downward motion and diabatic cooling at the lower level, whose boundaries are coherently located at 6-km height (Fig. 1d).

For the convenience of description in this study, the regions between radii of 40 and $80 \mathrm{~km}$ and outside $80 \mathrm{~km}$ are referred to as the inner-core and outer-core regions, respectively. Given that the stratiform precipitation outside the eyewall acts as a major contributor to lowlevel diabatic cooling, the inner-core diabatic cooling below 6-km height in the cloud microphysics package is artificially modified to examine the effect of increased diabatic cooling in the region of interest on SEF and ERC. In the primary sensitivity experiment (hereafter 

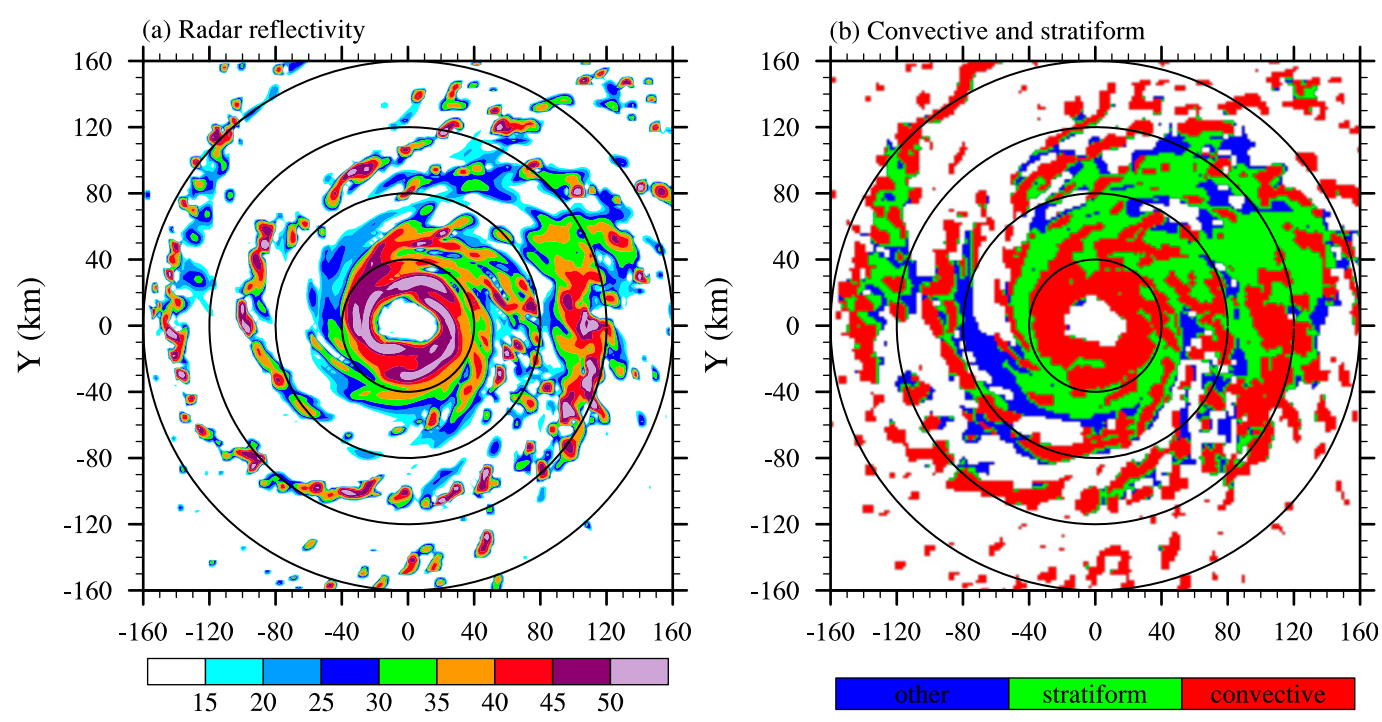

(c) $\mathrm{Vt}$ and $\mathrm{W}$
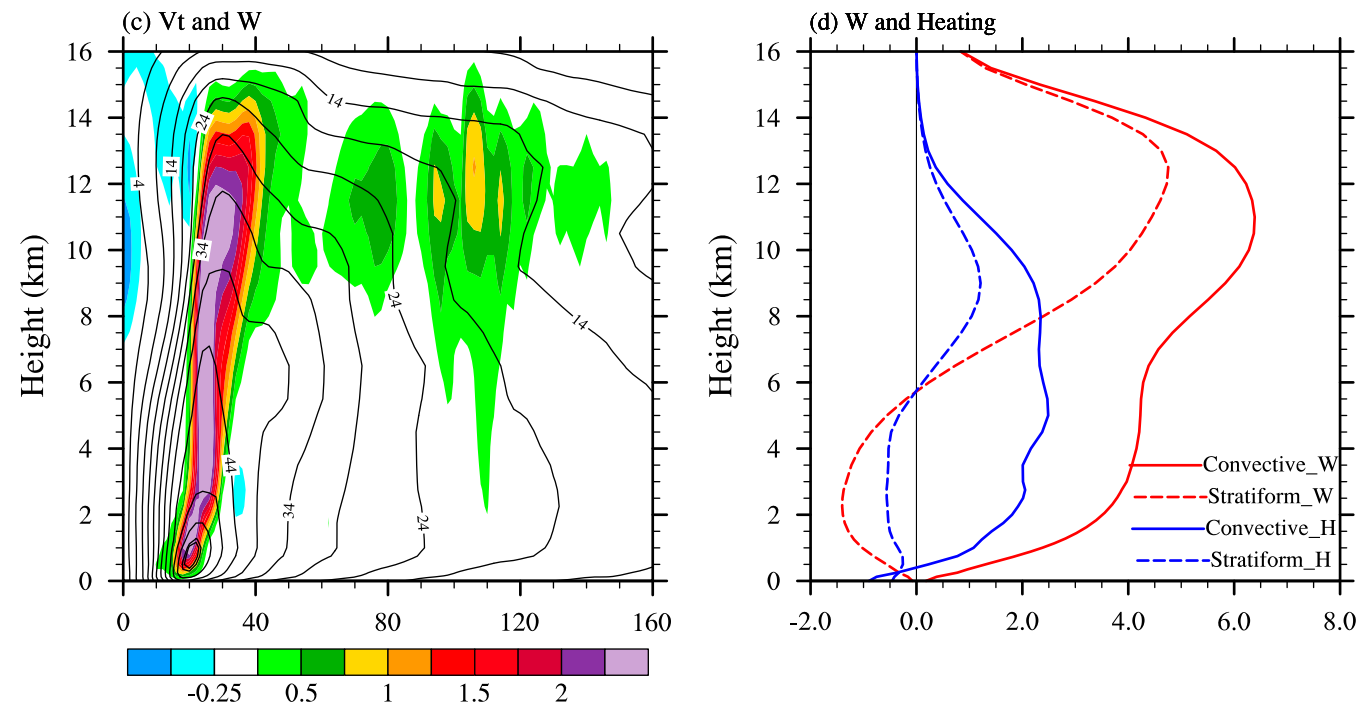

FIG. 1. Plan views of (a) simulated radar reflectivity $(\mathrm{dBZ})$ at $0.5-\mathrm{km}$ height and (b) different precipitation typesblue (other), (green) stratiform, and convective (red) - based on the partitioning algorithm of Rogers (2010); (c) radius-height cross section of azimuthal-mean tangential wind (contours, $\mathrm{m} \mathrm{s}^{-1}$ ) and vertical velocity (color shaded, $\mathrm{m} \mathrm{s}^{-1}$ ); (d) vertical profiles of vertical motion (red, $\times 10^{-1} \mathrm{~m} \mathrm{~s}^{-1}$ ) and diabatic heating (blue, $\times 10^{-3} \mathrm{~K} \mathrm{~s}^{-1}$ ) composited for the convective (solid) and stratiform (dashed) grids averaged between 40- and 80-km radii at $62 \mathrm{~h}$ of integration.

termed C30), the simulation is initialized at $62 \mathrm{~h}$ of the control experiment (CTL), but with the low-level innercore diabatic cooling (i.e., diabatic heating rate with less than zero) increased by $30 \%$ in the annulus of $40-80 \mathrm{~km}$ from the storm center. To diminish the edge effect due to the abrupt change near the lateral boundary of the annulus, the linear transition zones between radii of $30-40$ and $80-90 \mathrm{~km}$ are set. To further investigate the sensitivity to the strength and radial location of increased diabatic cooling, four extra sensitivity experiments are carried out, in which the diabatic cooling as modified in $\mathrm{C} 30$ is increased by $20 \%(\mathrm{C} 20)$ and $40 \%$ (C40), and shifted radially inward by $10 \mathrm{~km}$ (C30-IN) and outward by $10 \mathrm{~km}$ (C30-OUT).

In addition, a number of supplementary experiments are also performed to examine the sensitivity of TC structural evolution to the selection of microphysics scheme, restart time, and height of the top of modified diabatic cooling (not shown). Among the experiments with the microphysics parameterization switched to the Lin, WSM5, and Thompson scheme, respectively (although the detailed structural evolution differs from 
that in C30), all of the simulated storms feature the active outer rainbands and subsequent ERCs, eventually evolving into a storm with a relatively large-sized eye and wide eyewall, consistent with the result in C30 as shown later. If the modification of diabatic cooling starts from $48,54,66$, and $72 \mathrm{~h}$ of integration, there still exists the similar structural evolution as shown in C30. However, the discernible difference can be identified in that the occurrence time of SEF and ERC in the experiments with early restart time lag behind those in the experiments with late restart time, which could be due to a longer time taken to establish a favorable environment for SEF and ERC in a weaker vortex. If the top height of increased diabatic cooling is lowered to $2-\mathrm{km}$ altitude, the effect of inner-core diabatic cooling will be diminished. As a result, the storm continues intensifying, though at a slower rate than that in CTL, without the presence of SEF and ERC. By comparison, if the diabatic cooling in the whole column within the inner-core region is increased by $30 \%$, the storm structure displays a similar evolution as in $\mathrm{C} 30$, given that most of the diabatic cooling concentrates at the mid- to lower levels below $6 \mathrm{~km}$.

\section{Characteristics of TC evolution in CTL and C30}

Figure 2 shows the time evolution of the simulated MSLP and MTW at $0.5-\mathrm{km}$ height. The MSLP in the storm of CTL first experiences a rapid drop from 62 to $108 \mathrm{~h}$, achieving a minimum of $898 \mathrm{hPa}$. From $134 \mathrm{~h}$, the storm starts to slowly weaken until about $170 \mathrm{~h}$, followed by a steady evolution with a MSLP of $916 \mathrm{hPa}$ at the end of integration (Fig. 2a). In good correspondence with the MSLP, the MTW has an opposite evolution, with a maximum of $101 \mathrm{~m} \mathrm{~s}^{-1}$ and a steady state of about $82 \mathrm{~m} \mathrm{~s}^{-1}$ (Fig. 2b). In contrast, the storm in C30 halts the intensification and maintains a steady evolution until $122 \mathrm{~h}$, and then the MSLP falls slowly from 122 to $160 \mathrm{~h}$ followed by a rapid drop until $172 \mathrm{~h}$, and subsequently keeps nearly unchanged. The MTW in C30 has a slow decrease before $146 \mathrm{~h}$ and an increase afterward. Of interest is that there are two evident downward bulges around 148 and $180 \mathrm{~h}$, which arise from the two ERCs to be discussed later.

Figure 3 compares the evolution of the axisymmetric vertical velocity at $3-\mathrm{km}$ height and tangential wind at $0.5-\mathrm{km}$ height in CTL and C30. As the storm in CTL intensifies, the eyewall, indicative of ascending motion, gradually grows thick, which is accompanied by a slow increase in the TC inner-core size with the RMW increasing from 20 to $30 \mathrm{~km}$ through the simulation. After $100 \mathrm{~h}$, with the outward expansion and broadening of the eyewall, the rainbands outside the eyewall are

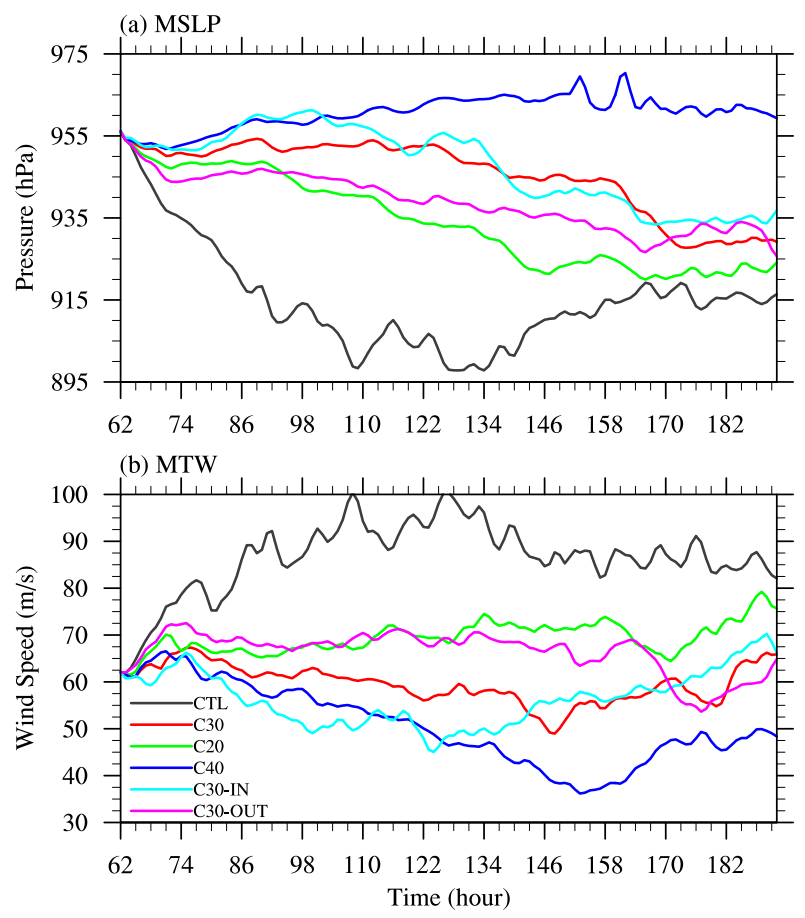

FIG. 2. Time evolution of (a) minimum sea level pressure (hPa) and (b) azimuthal-mean tangential wind at $0.5-\mathrm{km}$ height $\left(\mathrm{m} \mathrm{s}^{-1}\right)$ in all experiments: CTL (black). C30 (red), C20 (green), C40 (blue), C30-IN (cyan), and C30-OUT (purple).

significantly suppressed, leaving a wide region of downward motion. (Fig. 3a).

By comparison, the $\mathrm{TC}$ structure in $\mathrm{C} 30$ exhibits a quite different evolution, as illustrated in Fig. 3b. During the early stage between 63 and $100 \mathrm{~h}$, one distinct feature is that the convective activity appears to be relatively calm in the inner-core region, while the convective updrafts are discernible immediately outside $80 \mathrm{~km}$. After $100 \mathrm{~h}$, the outer-core rainbands become robust. As a response to the enhanced outer-core convective heating, the radius of hurricane-force wind $\left(\sim 33 \mathrm{~m} \mathrm{~s}^{-1}\right)$ starts to expand outward. Later on, the convective updrafts become vigorous within the inner-core region from $140 \mathrm{~h}$. In particular, at $148 \mathrm{~h}$, a strong concentric convective ring occurs at the radius of $50 \mathrm{~km}$, generating the first jump of RMW from 18 to $40 \mathrm{~km}$. Subsequently, the concentric convective ring contracts inward, and the primary eyewall gradually dissipates and is eventually replaced by the concentric convective eyewall, completing the first ERC. Concurrently, the convection in the outer region still remains vigorous. After $170 \mathrm{~h}$, one new strong and thick convective eyewall emerges at a radius of $55 \mathrm{~km}$. As a result, the inner eyewall collapses again and the RMW experiences the second jump from 25 to $50 \mathrm{~km}$, forming a TC structure of a large eye and a 


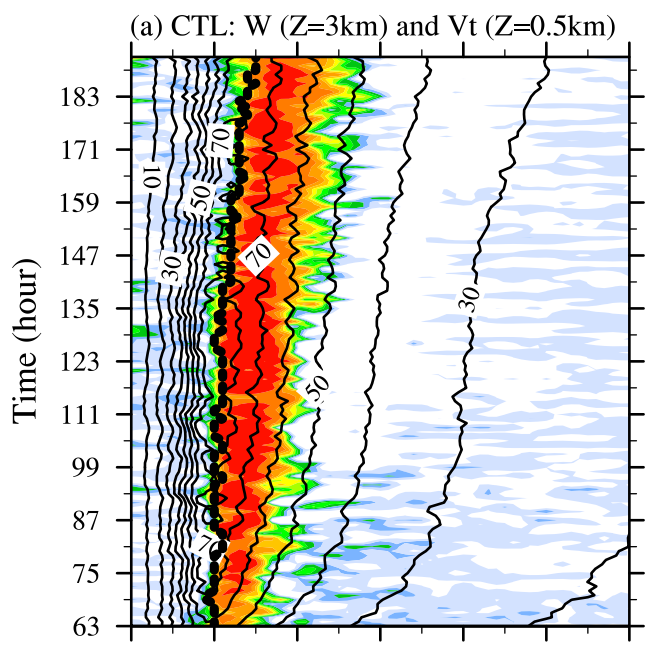

(c) CTL: Surface heat flux and wind speed

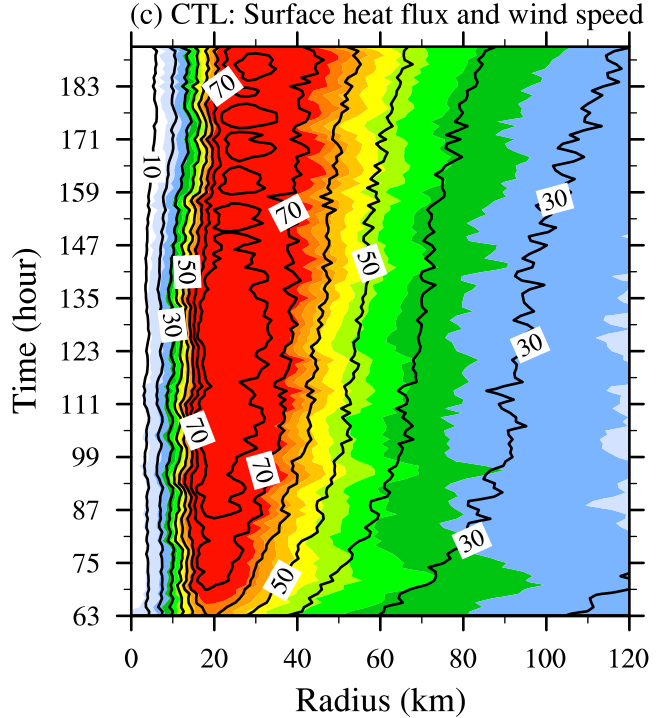

(b) $\mathrm{C} 30: \mathrm{W}(\mathrm{Z}=3 \mathrm{~km})$ and $\mathrm{Vt}(\mathrm{Z}=0.5 \mathrm{~km})$

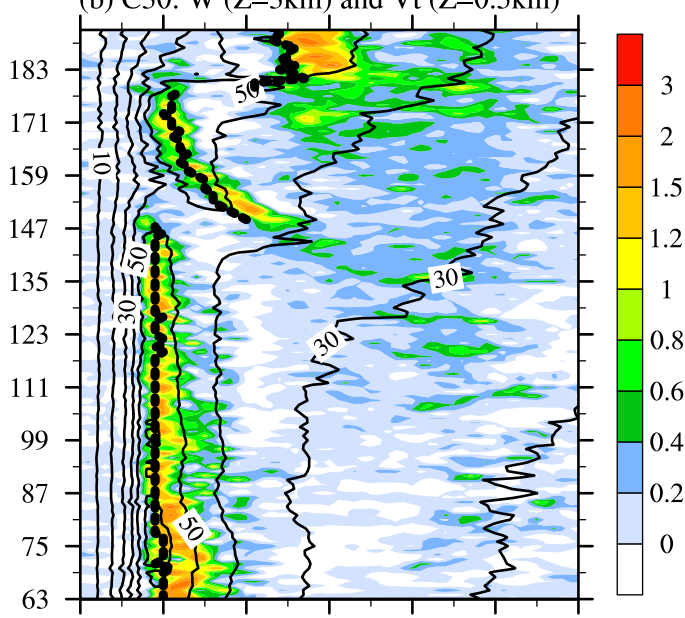

(d) C30: Surface heat flux and wind speed

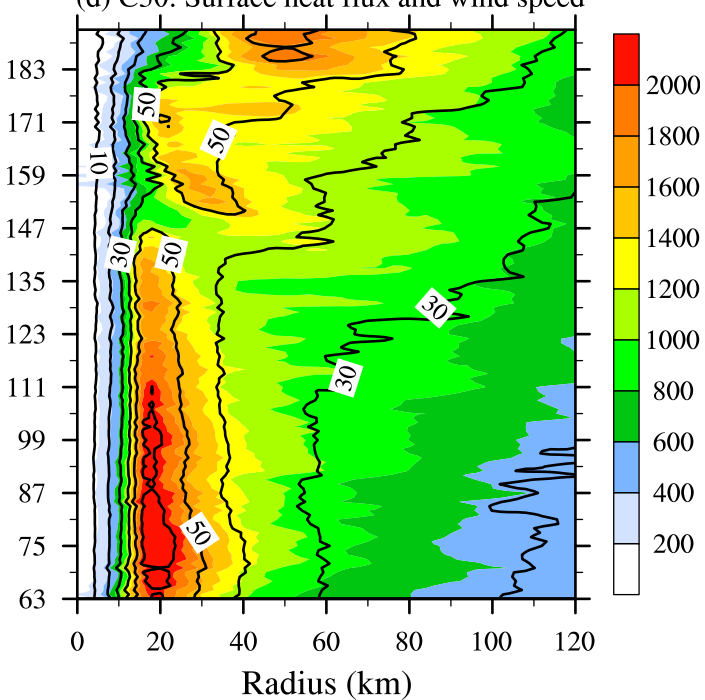

FIG. 3. Radius-time Hovmöller diagrams of (top) azimuthal-mean vertical velocity at 3-km height (color shaded,

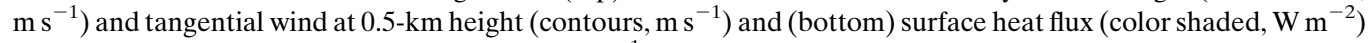
and near-surface total wind speed (contours, $\mathrm{m} \mathrm{s}^{-1}$ ) for (a),(c) CTL and (b),(d) C30. The thick black lines in (a),(b) denote the radius of maximum azimuthal-mean tangential wind at $0.5-\mathrm{km}$ height.

wide eyewall at the end of simulation bearing some resemblance to an annular hurricane (AH; Knaff et al. 2003; Wang 2008b; Zhou and Wang 2009). As exhibited in Figs. 3c,d, although the surface wind in C30 has a smaller speed than that in CTL during the early stage, the surface heat flux outside the primary eyewall is more expansive because of the enhanced air-sea thermal difference within the inner-core region. Prior to the first SEF at $148 \mathrm{~h}$, accompanied by the outward expansion of a strong wind field, the surface heat flux within the inner-core region is increased, which provides a favorable environment for the subsequent SEF.

As displayed in Fig. 4, snapshots of radar reflectivity at 3-km altitude in C30 shows that, prior to the first ERC, the outer rainbands are active outside $80 \mathrm{~km}$ (Fig. 4a), and then the well-organized inner spiral rainbands can be discernible after $139 \mathrm{~h}$. These inner spiral rainbands are axisymmetrized as they move inward and become tightly wound, facilitating the formation of a secondary convective ring (Figs. 4b-g). Afterward, the inner eyewall breaks down and is replaced by the inwardcontracting concentric convective ring (Figs. 4h-1). In the second ERC, a wide convective band is strengthened within the inner-core region, similarly accompanied by the collapse of the old inner eyewall. However, the difference is that the new outer convective band in the second ERC does not contract significantly, leading to a large-eyed storm (Figs. $4 \mathrm{~m}-\mathrm{t}$ ). The above-described 

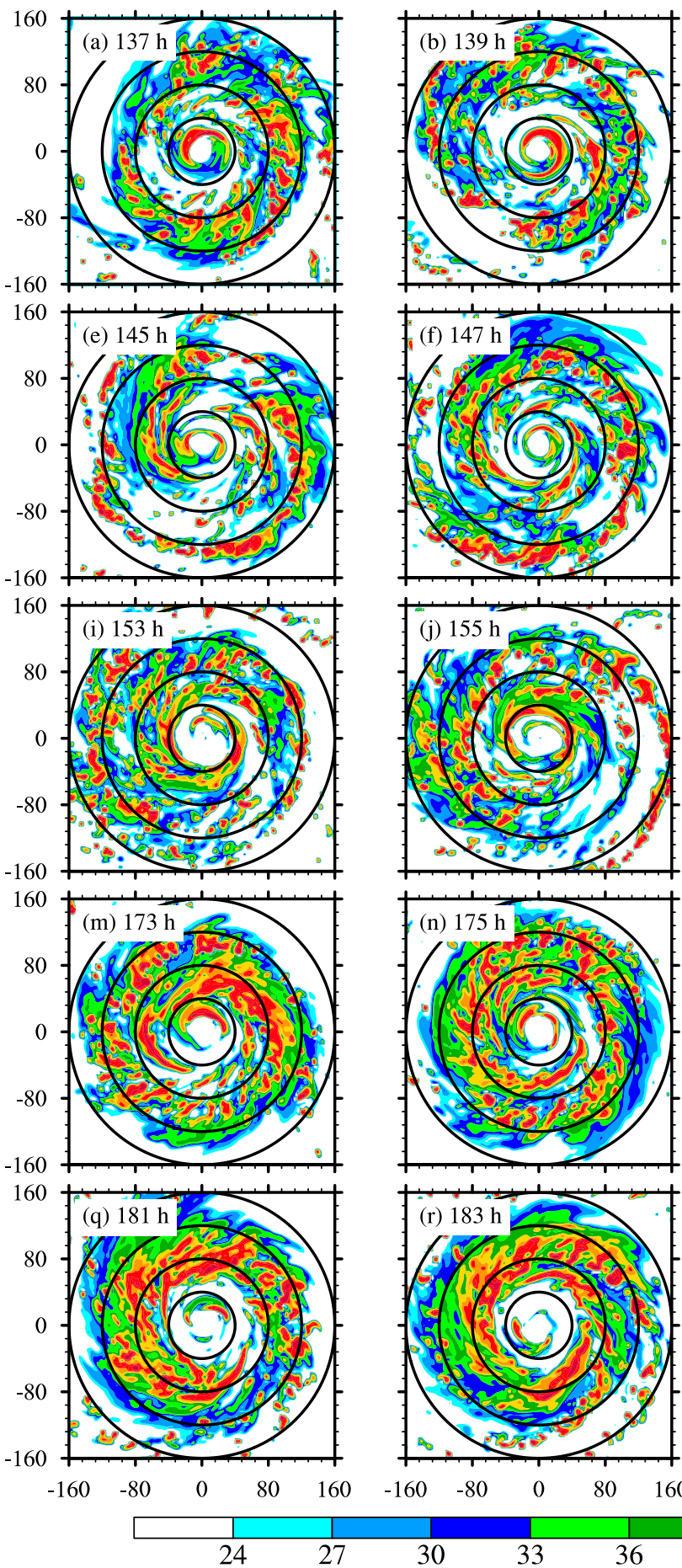

$\begin{array}{lllll}-160 & -80 & 0 & 80 & 160\end{array}$
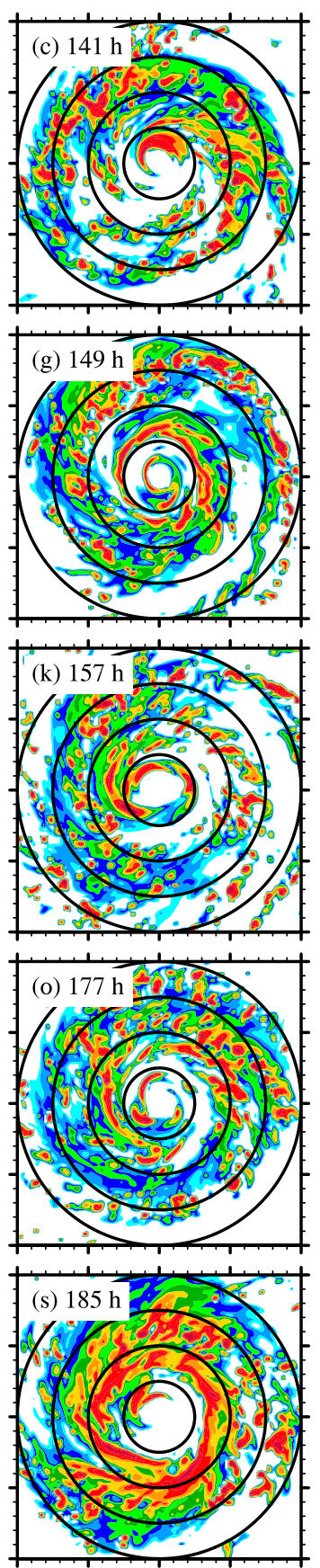

$\begin{array}{lllll}-160 & -80 & 0 & 80 & 160\end{array}$
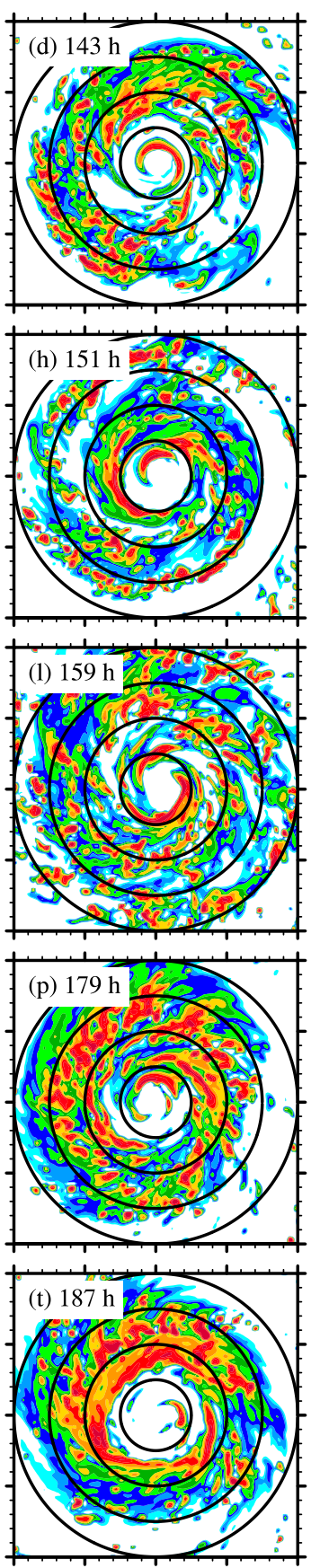

$\begin{array}{lllll}-160 & -80 & 0 & 80 & 160\end{array}$

FIG. 4. Snapshots of simulated radar reflectivity (dBZ) at 3-km height in $\mathrm{C} 30$ at different times from (a) $137 \mathrm{~h}$ to (t) $187 \mathrm{~h}$. The circles are placed at every $40-\mathrm{km}$ radius from the storm center.

evolution also supports the argument proposed previously that the concentric eyewall replacement is a route to the formation of an AH (e.g., Zhou and Wang 2009; Wang et al. 2013).
To elucidate the differences in convection activity within the inner- and outer-core regions between CTL and C30 during the early stage, the radial profiles of $\theta_{e}$, vertical velocity, radial wind, and divergence averaged 
(a) Equivalent potential temperature

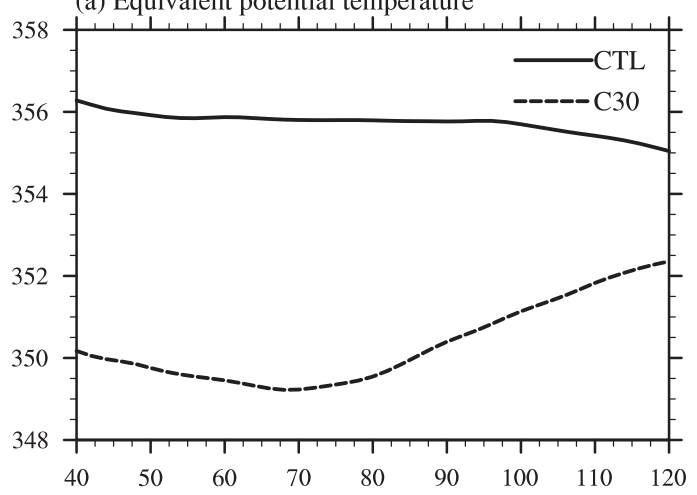

(c) Radial wind

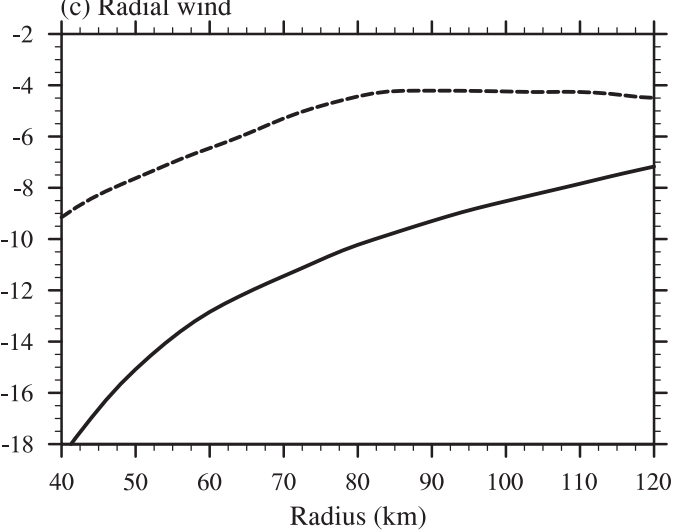

(b) Vertical velocity

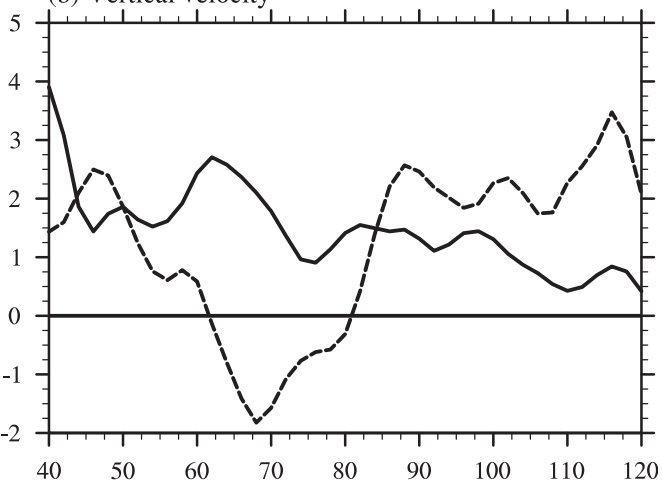

(d) Divergence

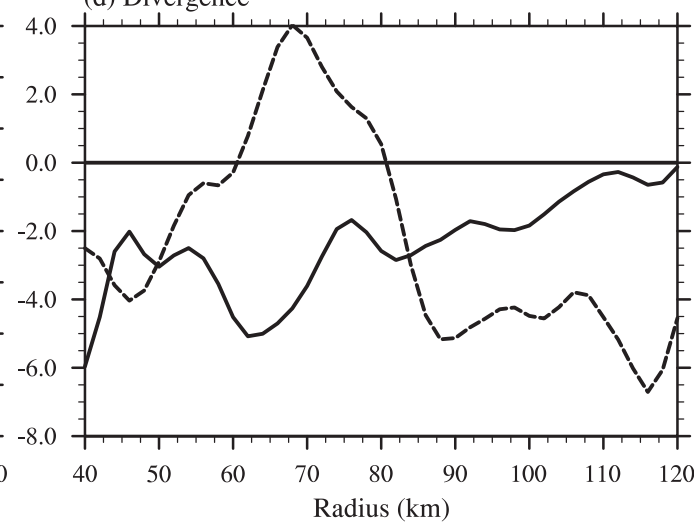

FIG. 5. Radial profiles of azimuthal-mean (a) equivalent potential temperature (K), (b) vertical velocity $\left(\times 10^{-2} \mathrm{~m} \mathrm{~s}^{-1}\right)$, (c) radial velocity $\left(\mathrm{m} \mathrm{s}^{-1}\right)$, and $(\mathrm{d})$ divergence $\left(\times 10^{-5} \mathrm{~s}^{-1}\right)$ at $1-\mathrm{km}$ height averaged from 70 to $90 \mathrm{~h}$ in $\mathrm{CTL}$ (solid line) and C30 (dashed line).

within the lowest $1-\mathrm{km}$ height from 70 to $90 \mathrm{~h}$ are shown in Fig. 5. The increased low-level diabatic cooling can largely lower the air entropy in the inner-core region, producing a large difference in $\theta_{e}$ between CTL and C30 (Fig. 5a). Along with the low-level inner-core air cooling, the reduced or negative buoyancy inhibits convective updrafts and even drives descending motion in the inner-core region in C30 (Fig. 5b). Dynamically, in response to the inner-core low-level cooling in $\mathrm{C} 30$, the elevated near-surface pressure would tend to reinforce the low-level divergence and thus descending motion within the inner-core region. Meanwhile, it also lessens the radial pressure gradient between the inner- and outer-core regions, facilitating the deceleration of radial inflow, thus convergence and upward motion outside $80 \mathrm{~km}$ (Figs. 5b-d). In some azimuthal sections, the near-surface outflows associated with the enhanced inner-core downdrafts are present in C30 (not shown). By comparison, the boundary layer in CTL features the coherent convergence that gradually weakens with increasing radius (Fig. 5d). The structure in C30 seems to bear some resemblance to that associated with the cold-pool dynamics (Didlake and Houze 2009; Sawada and Iwasaki 2010). For example, Sawada and Iwasaki (2010) found that, because of the convergence between the outflow associated with the cold pool and the low-level inflow, new convective cells can be initiated upstream of the preexisting surface cold pool triggered by evaporative cooling. These cells are finally organized into new spirally shaped outer rainbands.

Figure 6 depicts the diabatic heating and upward motion in the outer-core region, further supporting that there exist more vigorous outer rainbands in C30 than those in CTL. The progressive strengthening of the outer-core convection preceding the first ERC in C30 implies a positive feedback among convection, low-level convergence, and moisture flux. The enhanced outercore low-level convergence can favor the moisture convergence and increase the moist instability in the low troposphere, which is conducive to the convection development. In turn, the strengthened convection further fosters the moisture convergence and upward transport into the convection. The active outer-core rainbands 
(a) CTL: Heating and W

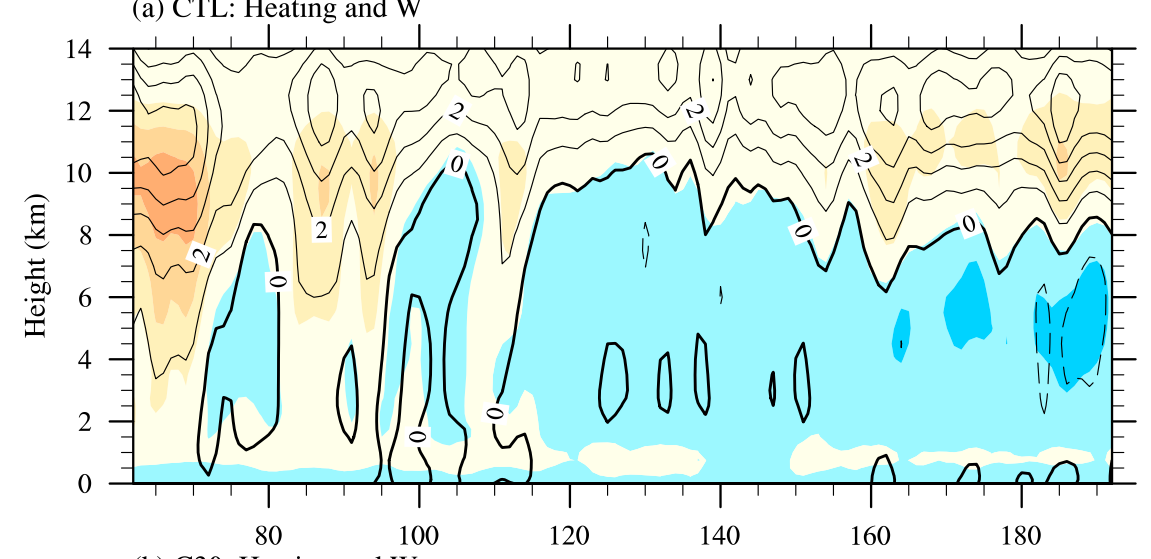

(b) C30: Heating and $\mathrm{W}$

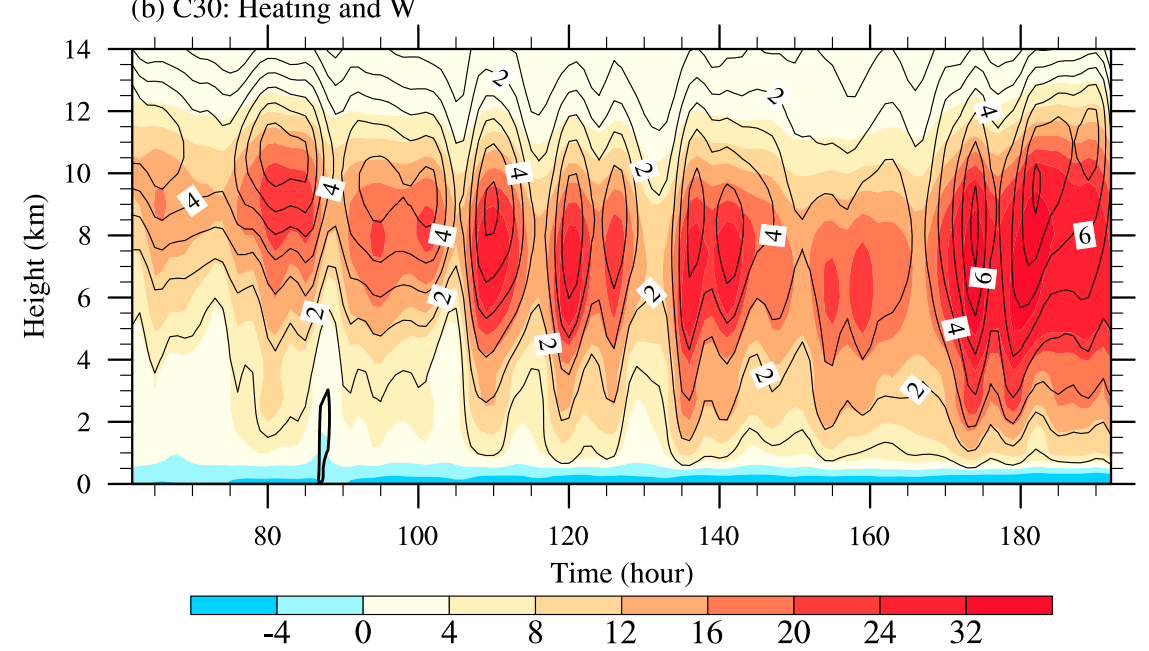

FIG. 6. Time-height cross sections of diabatic heating (color shaded, $\times 10^{-4} \mathrm{~K} \mathrm{~s}^{-1}$ ) and vertical velocity (contours, $\times 10^{-1} \mathrm{~m} \mathrm{~s}^{-1}$ ) averaged between 80 - and $120-\mathrm{km}$ radii in (a) CTL and (b) C30.

play a key role in the initiation of subsequent SEF, which will be substantiated in the following section.

To clearly show the SEF and ERC in C30, the heightradius cross sections of the azimuthal-mean diabatic heating, tangential wind, and transverse circulation are exhibited in Fig. 7. At $133 \mathrm{~h}$, the secondary maximum diabatic heating can be detected at a radius of $90 \mathrm{~km}$ (Fig. 7a), and subsequently becomes strengthened and moves inward entering into the inner-core region (Fig. 7b). Together with the enhanced outer convection, the vortex wind field has an apparent expansion in the low troposphere. Specifically, the $35 \mathrm{~m} \mathrm{~s}^{-1}$ contour of the tangential wind velocity at $1-\mathrm{km}$ height moves radially outward from 45 to $90 \mathrm{~km}$ between 133 and $139 \mathrm{~h}$ (Figs. 7a-c). One striking feature is that a local maximum diabatic heating builds up at about radius of $50 \mathrm{~km}$ starting from $142 \mathrm{~h}$, separating from the outer-core counterpart (Fig. 7d). Afterward, this strong diabatic heating extends vertically and forms a well-organized concentric convective ring, with the magnitude comparable to that in the inner eyewall (Figs. 7e,f). At $148 \mathrm{~h}$, a secondary tangential wind maximum emerges in the upper boundary layer at a radius of $40 \mathrm{~km}$, which is accompanied by the establishment of the secondary convective eyewall analogous to the collocation of peak wind and convection in the primary eyewall, marking a robust SEF. With the maturity and inward-contraction of the secondary eyewall, the primary eyewall and inner maximum tangential wind dissipate, whereas the secondary eyewall and corresponding maximum tangential wind assume the role of their predecessors (Figs. $7 \mathrm{~g}, \mathrm{~h}$ ).

During the second ERC, since the large outwardexpanding tangential winds persistently maintain at the low levels, the inner-core convection sustains robust with a wider radial extent in contrast to that in the first ERC (Figs. 7i-m). As the inner-core convection intensifies forming a new well-organized secondary eyewall, the primary eyewall decays and the maximum 

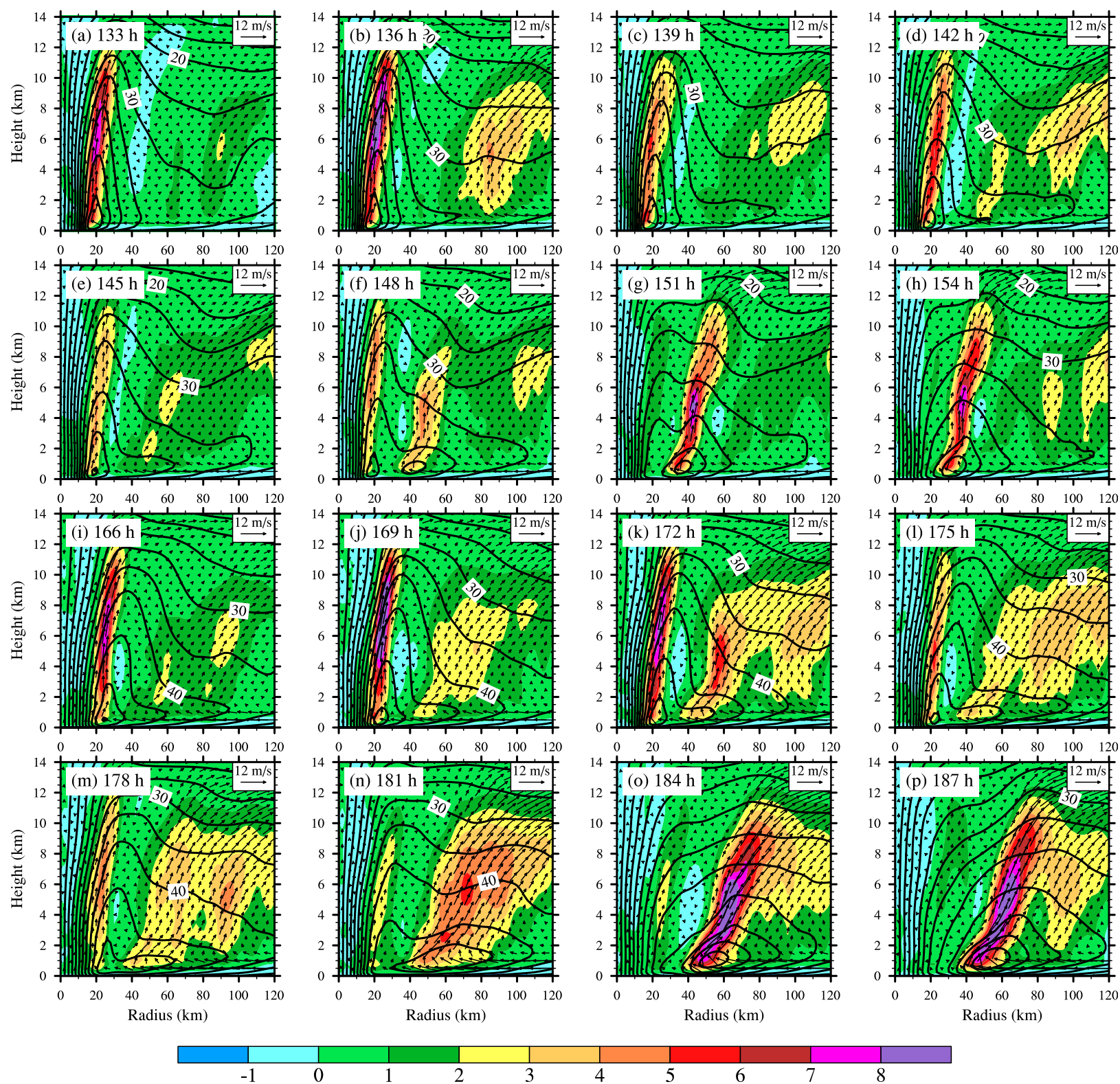

FIG. 7. Radius-height cross sections of azimuthal-mean diabatic heating (color shaded, $\times 10^{-3} \mathrm{~K} \mathrm{~s}^{-1}$ ), tangential wind (contours with intervals of $5 \mathrm{~m} \mathrm{~s}^{-1}$ ), and secondary circulation (arrows; $\mathrm{m} \mathrm{s}^{-1}$ with magnitude arrows at top right of each panel) at different times from (a) 133 to (p) $187 \mathrm{~h}$. For display purposes, the vertical velocity in the circulation vectors has been multiplied by a factor of 6 .

azimuthal-mean tangential wind is shifted outward to about $60 \mathrm{~km}$ (Fig. 7n). Ultimately, one strong and thick eyewall with a largely outward slope with height is established at a large radius (Figs. 7o,p). Similar to the $\mathrm{AH}$ structure discussed by Wang (2008b), the low-level tangential wind in response to the condensational heating in the outwardly tilted eyewall would be a decrease inside and an increase outside the RMW, which would prevent the eyewall from contracting and thus cause an increase in TC inner-core size. How the largely outward-tilted eyewall is formed will be discussed in section 5, together with the similar structure in C40.

\section{Dynamical mechanisms of SEF and ERC}

\section{a. Ambient dynamic parameters associated with $S E F$} and ERC

To evaluate the dynamical conditions favorable for SEF, three parameters, effective $\beta$, filamentation time, and inertial stability, are compared between 
CTL and C30. Their formulas can be expressed as $\beta=-(\partial \bar{q} / \partial r)\left(f_{0}+2 \bar{v} / r\right) / \bar{q}, \quad \tau_{\mathrm{fil}}=[-(\bar{v} / r) \partial \bar{v} / \partial r]^{1 / 2}, \quad$ and $I=\left\{\left(f_{0}+2 \bar{v} / r\right)\left[f_{0}+\partial(r \bar{v}) / r \partial r\right]\right\}^{1 / 2} / f_{0}$, respectively, in which $f_{0}$ is the Coriolis parameter at $18^{\circ} \mathrm{N}, q$ is the Ertel potential vorticity, $v$ is the tangential wind velocity, and $r$ is the radius from the TC center. The overbar represents the azimuthal-mean field. According to the BAS hypothesis proposed by Terwey and Montgomery (2008), a region of $\beta$ skirt with a weak but positive effective $\beta$, which facilitates the transfer of perturbation vorticity and kinetic energy from sporadic deep convection to azimuthal-mean flow, is required for SEF. Rozoff et al. (2006) pointed out that a strong filamentation associated with swirling tangential flows is more likely to shear apart nascent convective cells before they develop. As a result, moist convection is potentially suppressed in regions where the filamentation time is less than $30 \mathrm{~min}$, a characteristic value for deep convective overturning. Besides, with increasing inertial stability, the conversion of potential energy to kinetic energy and the warming of atmospheric column are more efficient (Shapiro and Willoughby 1982; Schubert and Hack 1982). Hence, for a given heat source, a region with higher inertial stability can provide a more beneficial dynamical condition to lower surface pressure and increase local wind speed.

Figure 8 displays the radius-height cross sections of three dynamic parameters averaged between 128 and $140 \mathrm{~h}$. The compact storm structure in CTL produces the large negative radial gradient of tangential wind $(\partial \bar{v} / \partial r<0)$ outside the primary eyewall, which leads to the small negative $\bar{q}(\bar{q}<0)$ near the surface at a radius of $50 \mathrm{~km}$ and $3-\mathrm{km}$ height at a radius of $80 \mathrm{~km}$, thus producing the large negative effective $\beta$ in the corresponding regions with the same sign of $\bar{q}$ and $\partial \bar{q} / \partial r$. It is apparent that the strong negative effective $\beta$ dominates the boundary layer outside $40 \mathrm{~km}$ and extends outward and upward in CTL, which does not quite support the SEF according to the BSA (Fig. 8a). In contrast, because of the active outer rainbands and outward-expanding wind field, the azimuthal-mean $\bar{q}$ in $\mathrm{C} 30$ is coherently positive with a certain magnitude. Therefore, although there are small negative effective $\beta$ values, the $\beta$ skirt can be identified in the lower troposphere outside $40 \mathrm{~km}$ in C30 (Fig. 8b). In terms of filamentation time, the outward expansion of the wind field in C30 can lessen the radial gradient of tangential wind (i.e., small negative $\partial \bar{v} / \partial r$ ), producing the filamentation time greater than $30 \mathrm{~min}$ in the inner-core region (Fig. 8d). In contrast, the relatively smaller filamentation time exists in the same region in CTL, because of the large negative $\partial \bar{v} / \partial r($ Fig. 8c). As a result, the large filamentation time in C30 is sufficient for the growth of convection in the inner-core region instead of it being disrupted by the straining process. Similarly, the large (small) negative $\partial \bar{v} / \partial r$ outside the primary eyewall in CTL (C30) is negative (positive) to the increase of inertial stability in the inner-core region (Figs. 8e,f). Rozoff et al. (2012) stated that the enhancement of inertial stability induced by the expanding wind field before the SEF can improve kinetic energy efficiency such that more convective latent heat is retained as kinetic energy. Overall, the $\beta$ skirt, long filamentation time, and high inertial stability in the inner-core region in $\mathrm{C} 30$ provide the dynamical conditions favorable for the SEF.

\section{b. Contribution of outer rainbands to SEF and ERC}

As described above, the outward expansion of the near-surface wind outside the primary eyewall can increase the surface entropy fluxes and generate the favorable environmental dynamical conditions facilitating the SEF. To quantitatively assess the individual contribution of the heating and momentum forcing from the primary eyewall and outer rainbands to the near-surface tangential wind tendency, the Sawyer-Eliassen (SE) equation as described in Bui et al. (2009) is adopted, which can be expressed as

$$
\begin{aligned}
\frac{\partial}{\partial r} & {\left[-g \frac{\partial \chi}{\partial z} \frac{1}{\rho r} \frac{\partial \psi}{\partial r}-\frac{\partial}{\partial z}(\chi C) \frac{1}{\rho r} \frac{\partial \psi}{\partial z}\right]+\frac{\partial}{\partial z}\left\{\left[\xi \chi(\zeta+f)+C \frac{\partial \chi}{\partial r}\right] \frac{1}{\rho r} \frac{\partial \psi}{\partial z}-\frac{\partial}{\partial z}(\chi C) \frac{1}{\rho r} \frac{\partial \psi}{\partial r}\right\} } \\
& =g \frac{\partial}{\partial r}\left(\chi^{2} Q\right)+\frac{\partial}{\partial z}\left(C \chi^{2} Q\right)-\frac{\partial}{\partial z}\left(\chi \xi F_{\lambda}\right)
\end{aligned}
$$

where $\psi$ is the streamfunction, $\chi=1 / \theta$ is defined as the reciprocal of potential temperature, $\xi=f_{0}+2 \bar{v} / r$ is the modified Coriolis parameter, $\zeta=\partial(r v) / r \partial r$ is the vertical component of relative vorticity, $C=v^{2} / r+f v$ is the sum of centrifugal and Coriolis force, and $Q$ and $F_{\lambda}$ represent the heating and momentum forcing, respectively. The WRF Model output variables are transformed into height and cylindrical polar coordinates. The variables $Q$ and $F_{\lambda}$ are taken as the residual terms of the azimuthal-mean thermodynamic and tangential momentum equations. 

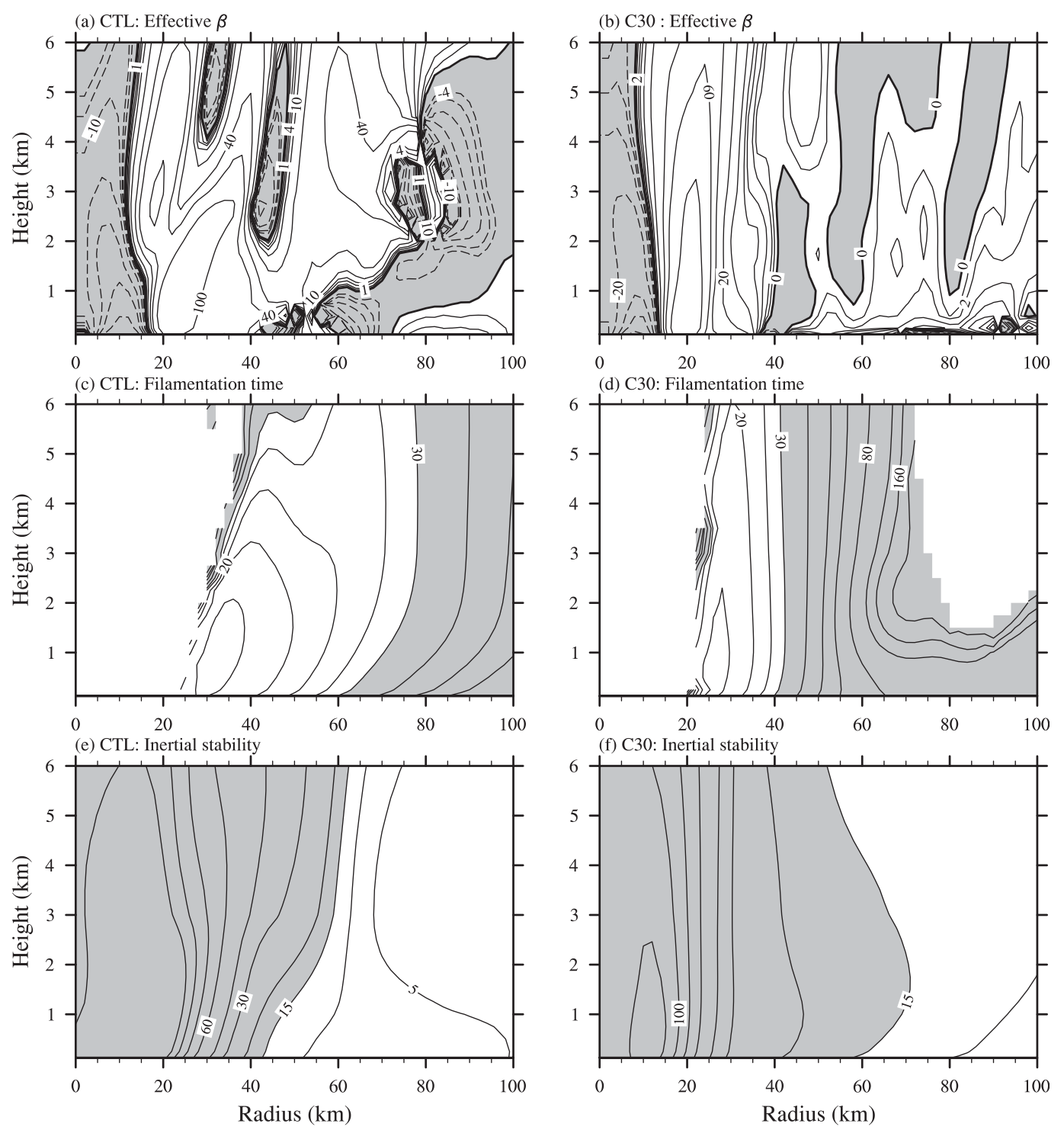

FIG. 8. Radius-height cross sections of (top) effective $\beta\left(\times 10^{-8} \mathrm{~m}^{-2} \mathrm{~s}^{-3}\right)$, (middle) filamentation time (min), and (bottom) inertial stability normalized by local Coriolis parameter averaged over 128 and $140 \mathrm{~h}$ in (left) CTL and (right) C30. (top to bottom) The shaded regions represent those with effective $\beta<0$, filamentation time $>30$ min, and normalized inertial stability $>15$, respectively.

Although both the hydrostatic and gradient wind balances in the SE equation generally hold invalid in the boundary layer, the SE diagnostics has been proven a useful tool in many previous studies, which still can be used to evaluate the individual contribution to the SEF from the heating and momentum forcing in the different regions in this study.

As a verification, the azimuthal-mean vertical and radial velocities at $136 \mathrm{~h}$ from the WRF simulation and the SE diagnostics are first compared. In Figs. 9a,b, it is evident that the vertical and radial velocities associated with both the primary and secondary eyewalls in WRF are reasonably captured by the SE model, such as the narrow convective updrafts and upper-level outflow in the primary eyewall, as well as the upward-extending outflow above the inflow boundary layer in the outer rainband region. However, some notable differences can also be found; for example, the magnitudes and heights of vertical and radial flows in the outer rainband region are smaller in the SE model than in WRF. In addition, there exist the strong radial inflows between the two upperlevel outflow channels in the SE output. These discrepancies may be attributed to the agradient (AW) wind balance and upper-level eddy effect (Bui et al. 2009; 

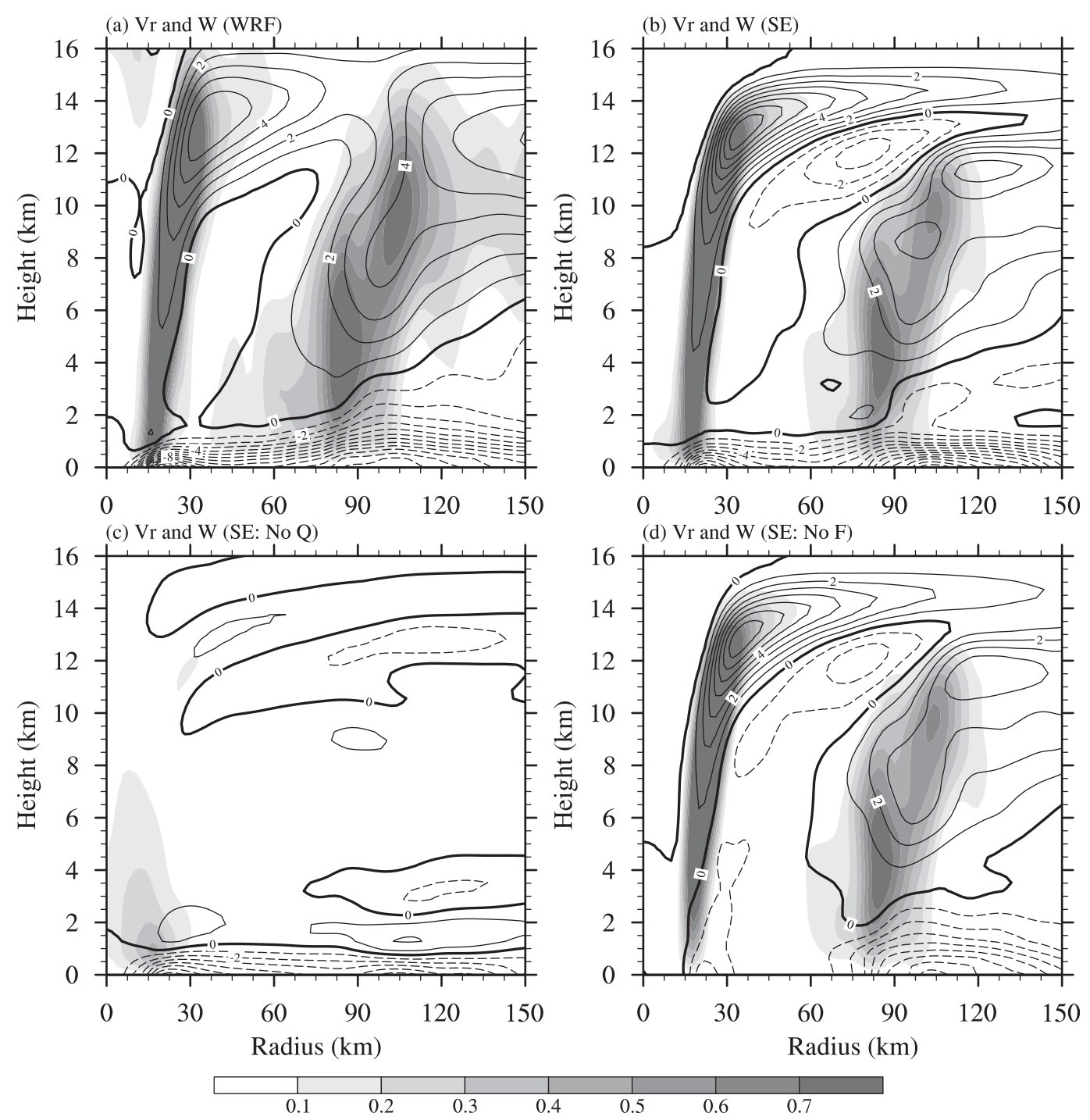

FIG. 9. Radius-height cross-sections of azimuthal-mean radial flow (contours, $\mathrm{m} \mathrm{s}^{-1}$ ) and vertical motion (gray shaded, $\mathrm{m} \mathrm{s}^{-1}$ ) in (a) WRF simulation and (b) SE model at $136 \mathrm{~h}$, and induced by (c) momentum forcing and (d) heating forcing in SE diagnostics, respectively.

Fudeyasu and Wang 2011). Nevertheless, the reasonable agreement of the results between the SE model and WRF assures the applicability for the purpose of this study. The merit of linear superposition of forcings on the right-hand side in the SE model allows us to evaluate the relative contribution of different forcings. In response to the momentum forcing, the radial inflows are dominant within the boundary layer because of the surface friction, and the vertical motion is only confined in the mid- to lower troposphere of the primary eyewall, accounting for a small amount of the total (Fig. 9c). In contrast, only given the heating forcing, the strong response of vertical motion corresponds well to the diabatic heating in the primary eyewall and outer rainband regions. The two lower-level inflow (upper-level outflow) maxima are located immediately outside both the convective eyewalls, respectively (Fig. 9d).

As can be seen in Fig. 10a, the WRF-simulated azimuthal-mean tangential wind tendency $(\partial \bar{v} / \partial t)$ at $1-\mathrm{km}$ height is positive in the inner-core region from $132 \mathrm{~h}$ through the first SEF. The maximum tangential wind tendency in the first ERC originates at a radius of $50 \mathrm{~km}$ around $144 \mathrm{~h}$, and then contracts inward accompanied by the shrinkage of the outer concentric convection. After the completion of the first ERC, the positive tangential wind tendency occupies most of the inner-core region and is progressively strengthened; while the tangential wind tendency in the inner eyewall 

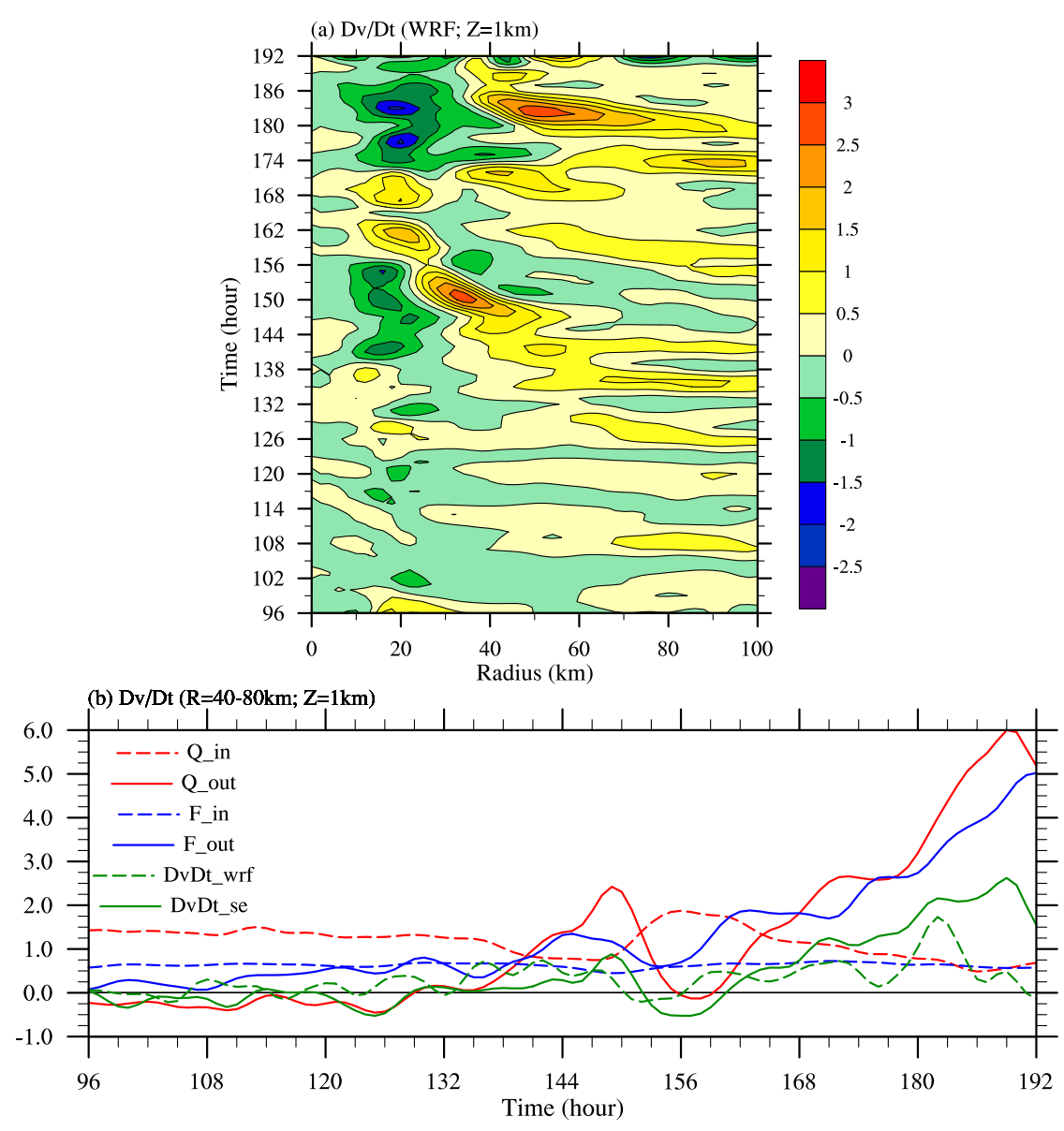

FIG. 10. (a) Radius-time Hovmöller diagram of azimuthal-mean tangential wind tendency at 1-km height (color shaded, $\mathrm{m} \mathrm{s}^{-1}$ ). (b) Time evolution of tangential wind tendency at 1-km height averaged between radii of $40-80 \mathrm{~km}$ induced by diabatic heating (red) and momentum (blue) in the primary eyewall region inside $40 \mathrm{~km}$ (dashed) and the outer rainband region outside $40 \mathrm{~km}$ (solid), and obtained from WRF (green dashed) and derived from the SE model based on Eq. (2) (green solid).

region is decreased until $183 \mathrm{~h}$ when the outer tangential wind culminates in the formation of a largeeyed storm. To evaluate the relative contribution to the tangential wind tendency from the heating and momentum forcings in the different regions, the azimuthal-mean radial velocity $(\bar{u})$ and vertical velocity $(\bar{w})$, forced by the heating and momentum in the primary eyewall region (inside $40 \mathrm{~km}$ ) and outer region (outside $40 \mathrm{~km}$ ), are diagnosed respectively, based on the streamfunction $(\psi)$ calculated from the $\mathrm{SE}$ equation. Then, the diagnosed transverse circulation $(\bar{u}$ and $\bar{w})$ is used to derive the acceleration of tangential wind based on the equation of axisymmetrized tangential wind tendency:

$$
\frac{\partial \bar{v}}{\partial t}=-\bar{u} \overline{\varsigma_{a}}-\bar{w} \frac{\partial \bar{v}}{\partial z}+\overline{F_{\lambda}}
$$

where $\overline{s_{a}}$ and $\overline{F_{\lambda}}$ are the azimuthal-mean absolute vorticity and radial momentum forcing, respectively. As displayed in Fig. 10b, the resultant tangential wind tendency at 1-km height averaged from 40 to $80 \mathrm{~km}$ derived from the sum of three terms on the right-hand side in Eq. (2) has roughly similar evolution to the counterpart in the WRF simulation (green solid and dashed lines), which captures the two episodes of enhanced inner-core tangential wind tendency corresponding to the two ERCs. However, we note that partly because the assumption of the gradient wind balance in the SE equation is not robust and the eddy effect is ignored in Eq. (2), the magnitude and phase of the tangential wind tendency derived from the SE diagnostics to a certain degree differ from those in the WRF simulation.

On the other hand, the tangential wind tendency derived from the transverse circulation $\left(-\bar{u} \overline{\varsigma_{a}}-\bar{w} \partial \bar{v} / \partial z\right)$ 
induced by the heating and momentum in the primary eyewall region and outer region is also exhibited in Fig. 10b. It indicates that, since the outer rainbands are active outside $80 \mathrm{~km}$ before $135 \mathrm{~h}$, the heating forcing from the inner eyewall makes a major positive contribution to the increase of tangential wind in the innercore region, but with a small magnitude. After $135 \mathrm{~h}$, accompanied by the inward extension of the outer rainbands, the contribution from the outer convective heating begins to rise and then surpasses the counterpart from the inner eyewall heating, reaching the first peak at $149 \mathrm{~h}$ (red solid and dashed lines). As the ERC proceeds, the secondary convective ring moves inside $40 \mathrm{~km}$ and its related contribution declines. After $160 \mathrm{~h}$, the outer rainbands reinvigorate, again resulting in a robust increase of inner-core tangential wind. Along with the variation of tangential wind outside $40 \mathrm{~km}$, the surface friction in the inner-core region also undergoes a nearly synchronous evolution. The inner-core tangential wind tendency solely induced by the momentum forcing in the outer region has a comparable magnitude to that induced by the heating forcing in the outer region, while the contribution from the momentum forcing in the primary eyewall region remains small throughout the simulation (blue solid and dashed lines). To sum up, although the primary eyewall heating makes a certain contribution to the expansion of low-level tangential wind during the early stage of simulation, as the outer rainbands contract inward, the enhanced heating and momentum forcing in the outer region contributes predominantly to the strengthening of the inner-core lowlevel tangential wind, promoting the SEF.

\section{c. Dynamical features in the boundary layer}

As noted in the introduction, Huang et al. (2012) pointed out that the unbalanced boundary layer dynamics associated with supergradient winds and positive agradient forces plays an essential role in the SEF. To measure the extent of the deviation from the gradient wind balance during the two ERCs, the agradient force (AF) and agradient wind (AW) are given as follows:

$$
\begin{aligned}
\mathrm{AF} & =-\frac{\partial \bar{p}}{\bar{\rho} \partial r}+f \bar{v}+\frac{\bar{v}^{2}}{r}, \\
\mathrm{AW} & =\bar{v}-\bar{v}_{g}
\end{aligned}
$$

where $p$ is pressure, $\rho$ is the air density, and $v_{g}$ is the gradient wind. The conditions $\mathrm{AF}>0$ or $\mathrm{AW}>0(\mathrm{AF}<$ 0 or AW $<0$ ), corresponding to a supergradient (subgradient) wind, indicates a tendency to decelerate (accelerate) the inflows. As exhibited in Figs. 11a,b, the time-height cross sections of AF and AW in the SEF region have the nearly same distribution with the maximum centers located at $1-\mathrm{km}$ height. Overall, the AF at the levels below $0.5 \mathrm{~km}$ is directed radially inward, corresponding to the shallow layer of subgradient winds, while the radially outward AF (supergradient winds) occupies the levels between 0.5 and $2 \mathrm{~km}$. Starting from $136 \mathrm{~h}$, each enhancement of AF and AW is collocated with the initiation of the concentric eyewall. In contrast to the first ERC episode, the AF and AW during the second ERC have larger and vertically deeper positive values. Within the boundary layer, the radial inflows are strengthened during the SEF, while the two outflow maxima located at $2-\mathrm{km}$ height are coincident with the two ERCs, reflecting the unbalanced outflow caused by the supergradient flow (Fig. 11b).

In Figs. 11c,d, it is apparently demonstrated that the radius-time evolutions of $\mathrm{AF}$ and $\mathrm{AW}$ within the boundary layer well correspond to that of low-level vertical motion as shown in Fig. 3b, namely, the wax and wane of the primary and secondary eyewalls keep pace with those of AF and AW. Prior to the two ERC episodes, the large inflows emerge just outside the maximum $\mathrm{AF}$ and $\mathrm{AW}$, which causes the inflow deceleration on the inward side of the maximum inflow, generating the convergence in the maximum AF and AW region. As a result, the progressive strengthening of boundary layer inflow and the radially outward $\mathrm{AF}(\mathrm{AF}>0)$ therein leads to an eruption of moist air out of the boundary layer to initiate and sustain deep convection for the SEF. The dynamic structure described here is well consistent with the study of Huang et al. (2012), who observed the unbalanced boundary layer dynamics characterized by supergradient wind and positive agradient force acting to initiate the SEF in collaboration with the increase in the boundary layer inflow.

The other alternative boundary layer theory of SEF is proposed by Kepert (2013), who emphasized that the key point for SEF is the development of a local enhancement of the radial vorticity gradient. Based on three diagnostic boundary layer models, he concluded that a small local increase in the radial gradient of vorticity at the outer radii can initiate the SEF through a positive feedback among the local enhancement of the radial vorticity gradient, the frictional updraft, and convection. Figure 12 exhibits the radial profiles of tangential wind and relative vorticity during the ERCs. With the outward expansion of tangential wind and the enhancement of surface heat flux in the inner-core region, the local vorticity maxima outside the primary eyewall can be discernible between the radii of 30 and $40 \mathrm{~km}$ at 139,142 , and $145 \mathrm{~h}$ before the first SEF (Figs. 12a,b). The strong convective updrafts occur near the radius of $50 \mathrm{~km}$ (Figs. 7c-e), which is also consistent 


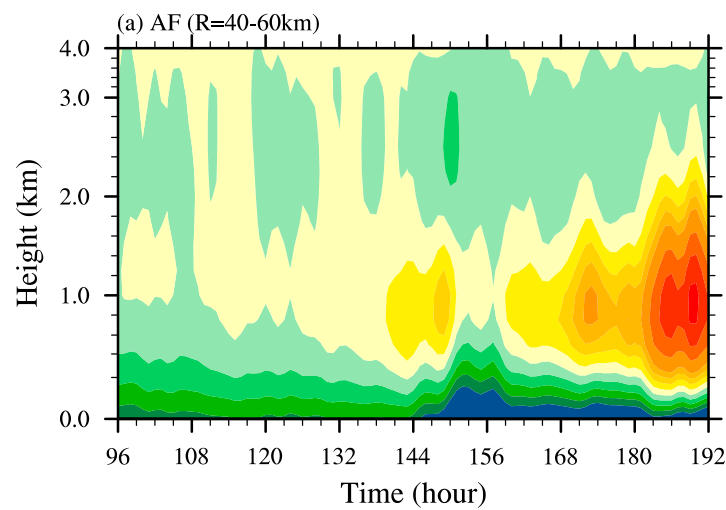

(c) $\mathrm{AF}(\mathrm{Z}=1 \mathrm{~km})$

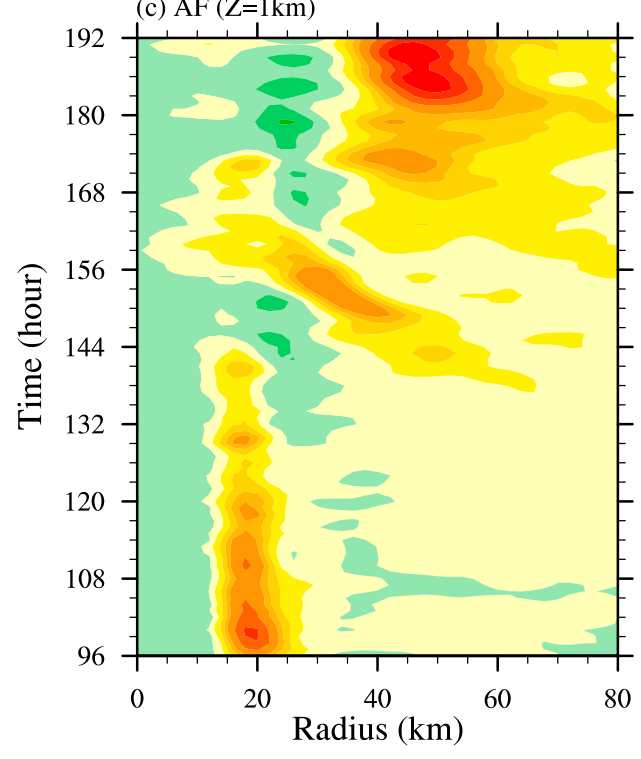

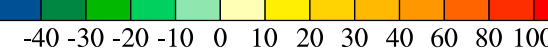

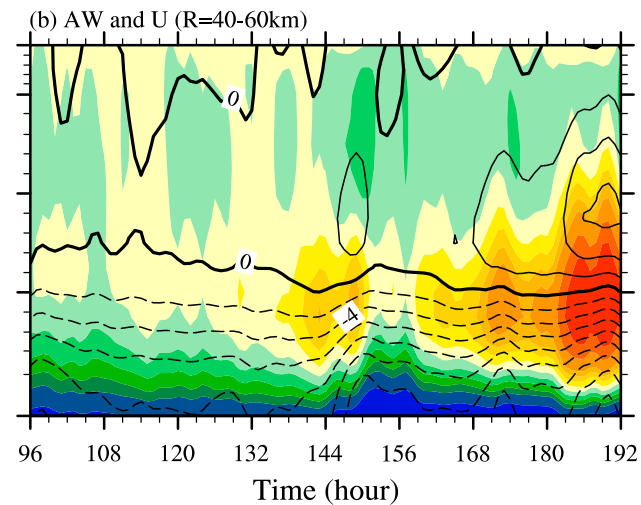

(d) AW $(\mathrm{Z}=1 \mathrm{~km})$ and $\mathrm{U}(\mathrm{Z}=0.25 \mathrm{~km})$
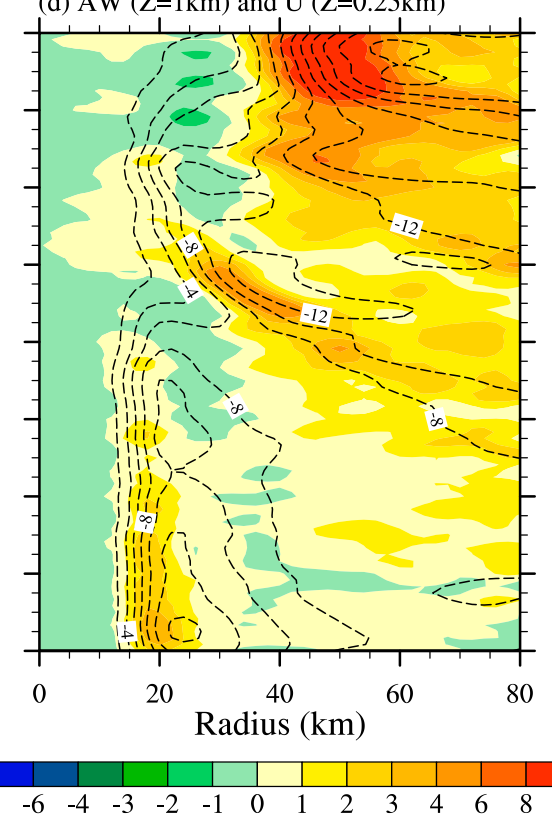

FIG. 11. Time-height cross sections of azimuthal-mean (a) agradient force (color shaded, $\mathrm{m} \mathrm{s}^{-1} \mathrm{~h}^{-1}$ ) and (b) agradient wind (color shaded, $\mathrm{m} \mathrm{s}^{-1}$ ) and radial wind (contours, $\mathrm{m} \mathrm{s}^{-1}$ ) averaged between the radii of 40 and $60 \mathrm{~km}$ in C30. Radius-time Hovmöller diagrams of azimuthal-mean (c) 1-km agradient force (color shaded, $\mathrm{m} \mathrm{s}^{-1} \mathrm{~h}^{-1}$ ) and (d) $1-\mathrm{km}$ agradient wind (color shaded, $\mathrm{m} \mathrm{s}^{-1}$ ) and $0.5-\mathrm{km}$ radial wind (contours, $\mathrm{m} \mathrm{s}^{-1}$ ). The completion of the first and second SEF occurs around 148 and $172 \mathrm{~h}$, respectively.

with Kepert's argument that the local maximum of axisymmetric vorticity outside the eyewall can lead to a significant local updraft on its outer edge, considering that the frictionally forced updraft is approximately proportional to the squared reciprocal of the absolute vorticity, the radial gradient of vorticity, and the wind speed squared [refer to his Eq. (24) in Kepert (2013)]. With time a secondary tangential wind maximum emerges corresponding to the first SEF at $148 \mathrm{~h}$, and then the inner eyewall vanishes, shifting the RMW outward to $38 \mathrm{~km}$. Similarly, during the second SEF, the local maximum vorticity and radial vorticity gradient can also be identified outside the primary eyewall (Figs. 12c,d), but with the radial location being farther outward, facilitating the second SEF at a relatively larger radius at $172 \mathrm{~h}$ (Fig. 7k). It should be pointed out that, distinct from the pure boundary layer models in Kepert (2013), the WRF simulation with full physics fails to quantitatively evaluate the relative contribution in association with the unbalanced boundary layer dynamics by Huang et al. (2012) and the boundary layer theory by Kepert (2013). However, careful examination of the occurrence time and radial extent of $\mathrm{AF}$ and vorticity manifests that the discernible positive $\mathrm{AF}$ emerges earlier and extends farther radially outward than the positive vorticity prior to the SEF in C30 (not shown). This implies that the unbalanced boundary layer dynamics may play a major role in triggering the 
(a) Vt (First ERC; SEF $=148 \mathrm{~h}$ )

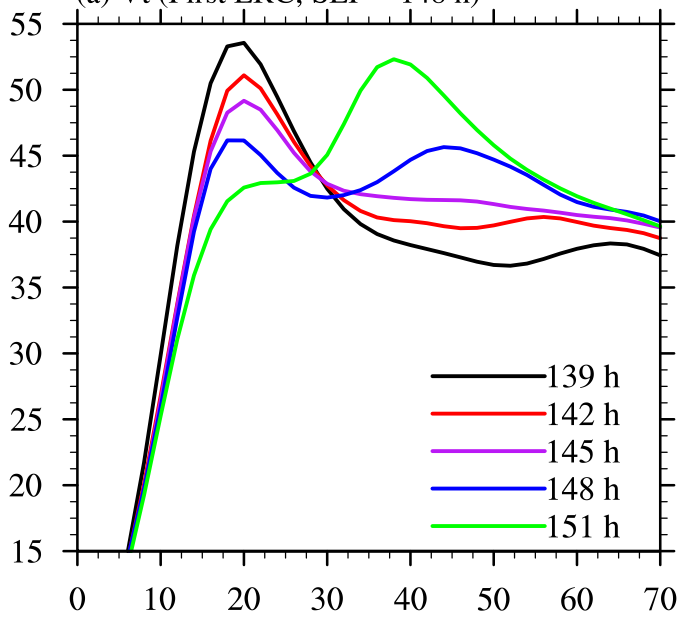

(c) Vt $($ Second ERC; SEF $=172 \mathrm{~h})$

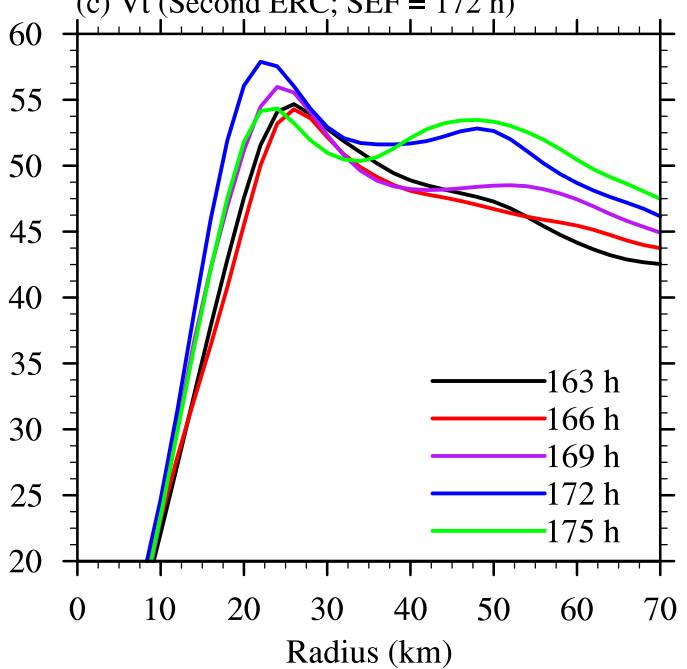

(b) Vorticity (First ERC; SEF $=148$ h)

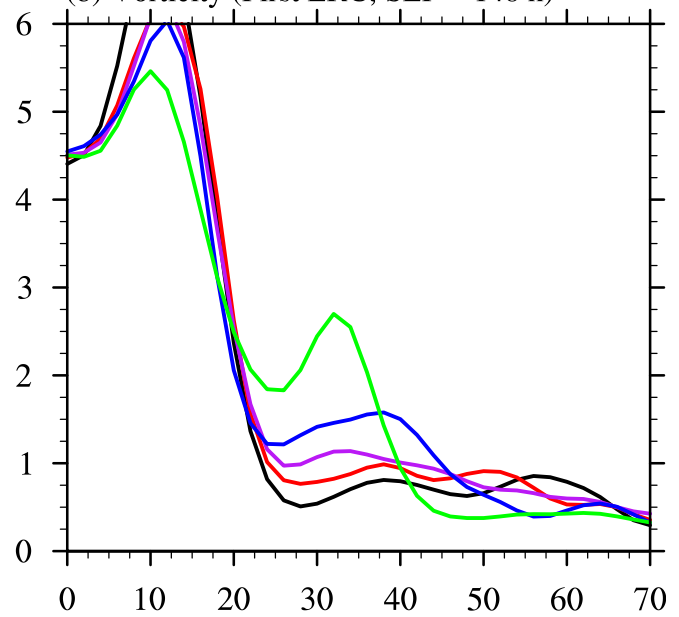

(d) Vorticity (Second ERC; SEF $=172 \mathrm{~h}$ )

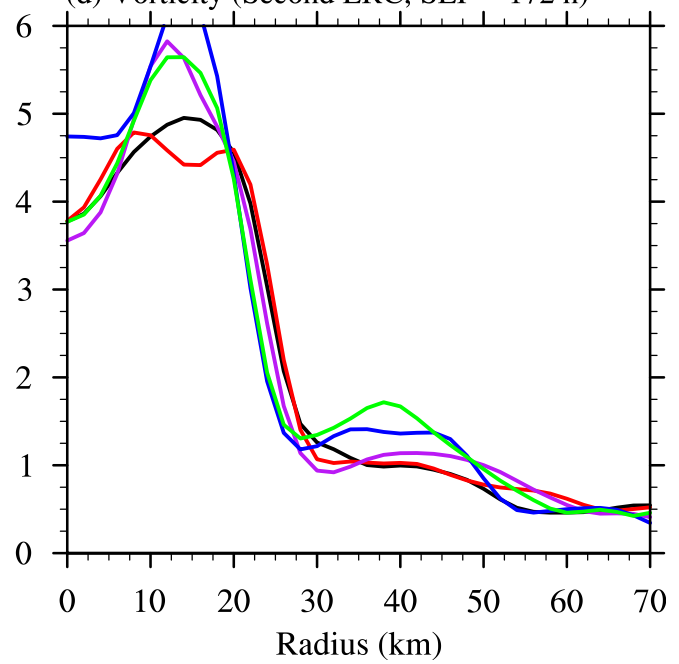

FIG. 12. Radial profiles of azimuthal-mean (a),(c) tangential wind $\left(\mathrm{m} \mathrm{s}^{-1}\right)$ and (b), (d) relative vorticity $\left(\times 10^{-3} \mathrm{~s}^{-1}\right)$ at 1-km height in different times during (a),(b) the first and (c),(d) the second ERC. The completion of the first and second SEF occurs around 148 and $172 \mathrm{~h}$, respectively.

SEF. Subsequently, as the positive vorticity grows in the SEF region, the boundary layer process proposed by Kepert (2013) commences to take effect, cooperatively contributing to the SEF.

\section{Sensitivity to strength and radial location of diabatic cooling}

\section{a. The behaviors in the extra sensitivity experiments}

To examine the impact of strength and radial location of the increased low-level cooling on the storm structure and intensity change, the extra four sensitivity experiments, namely, C20, C40, C30-IN, and C30-OUT, are also conducted, respectively. It can be seen clearly in Fig. 2 that the less increased low-level inner-core cooling in C20 achieves the strongest storm among all the sensitivity experiments, with the steady MSLP of about $920 \mathrm{hPa}$ at the end of the simulation. The azimuthalmean structural evolution in Fig. 13a illustrates that, although several features of secondary convective ring appear episodically immediately outside the primary eyewall in $\mathrm{C} 20$, there are no distinct ERCs, moat regions, and corresponding secondary tangential wind maxima, because the secondary convective rings are very close to the eyewall. After $162 \mathrm{~h}$, the convective updrafts within the radii of $40-60 \mathrm{~km}$ become robust, forming a large-eyed storm similar to the counterpart in C30. In contrast, the weakest storm is observed in C40 with the more increased low-level inner-core cooling. Note that the storm weakens persistently until about 
(a) $\mathrm{C} 20$

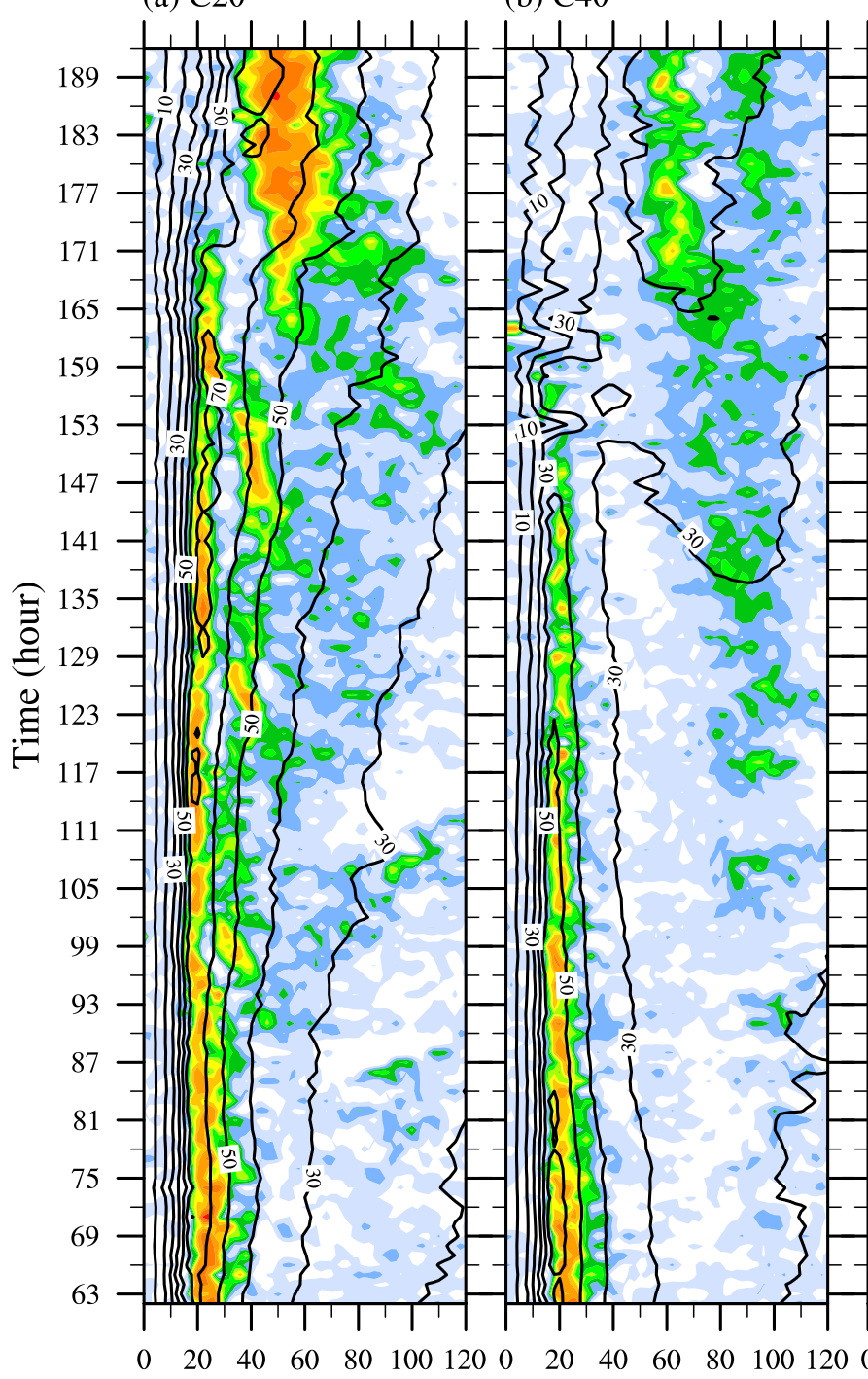

(c) C30-IN (d) C30-OUT

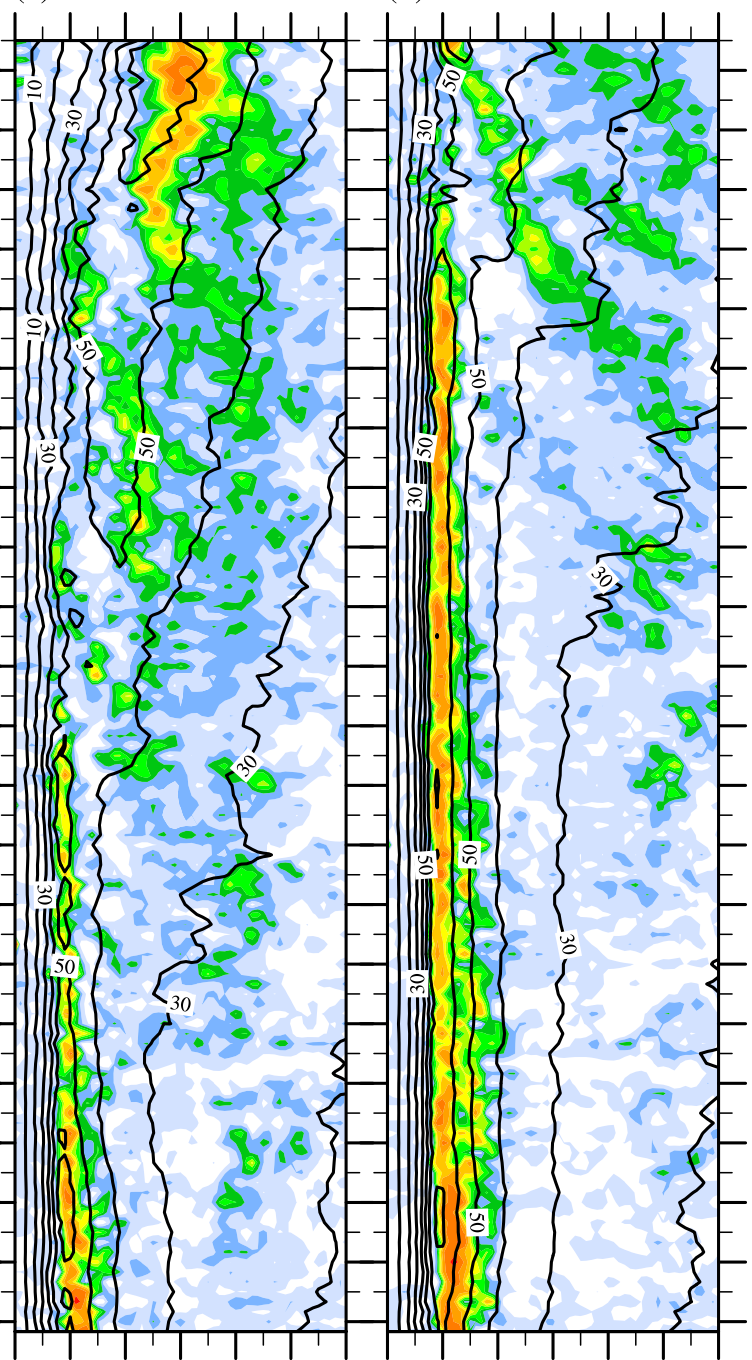

Radius (km)

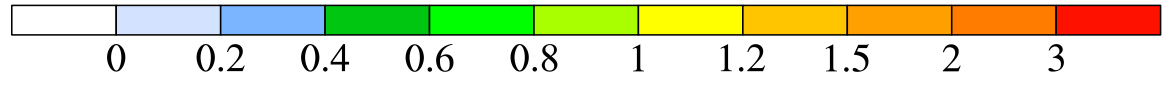

FIG. 13. As in Fig. 3, but in (a) C20, (b) C40, (c) C30-IN, and (d) C30-OUT.

$155 \mathrm{~h}$, and then starts to progressively intensify (Fig. 2), which is collocated with a distinct ERC in which the concentric rainbands originate outside a radius of $90 \mathrm{~km}$ (Fig. 13b). One unique feature in $\mathrm{C} 40$ is that the outer convective ring fails to contract to the radius of the primary eyewall, but rather resides and develops in the inner-core region. When shifting the increased low-level cooling radially inward by $10 \mathrm{~km}$, the storm in C30-IN has a pronounced decay before $100 \mathrm{~h}$, followed by an intensity oscillation and then a notable intensification after $128 \mathrm{~h}$ (Fig. 2). The convection in the primary eyewall fades quickly in C30-IN (Fig. 13c). The rainband activity mainly lies inside a radius of $80 \mathrm{~km}$, close to the primary eyewall region, in such a way that the outward expansion of low-level tangential wind occurs early. Specifically, the $30 \mathrm{~m} \mathrm{~s}^{-1}$ contour of azimuthal-mean tangential wind apparently expands outward from $96 \mathrm{~h}$, the earliest among all the experiments. For the storm in C30-OUT with a radially outward displacement of the increased low-level cooling, the MSLP has a slow drop and the MTW nearly keeps constant prior to $164 \mathrm{~h}$, suggesting that the outward displacement of the enhanced cooling lessens the detrimental influence on TC intensity. After $164 \mathrm{~h}$, there exists a discernible filling of 
(a) $\mathrm{C} 20$

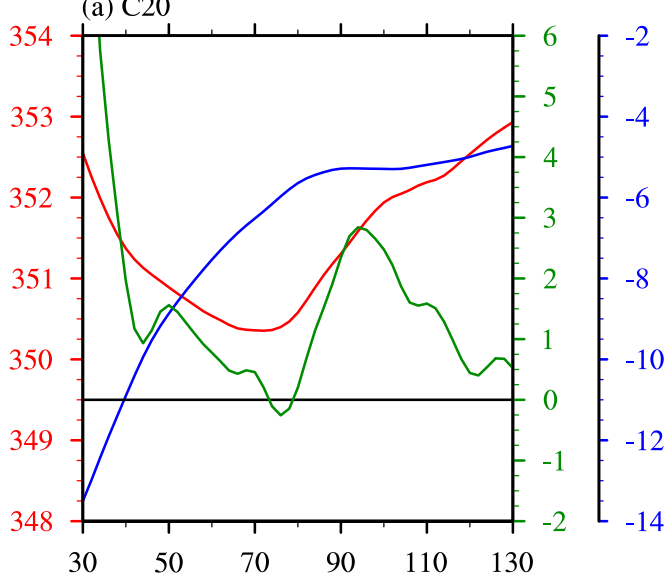

(c) $\mathrm{C} 30-\mathrm{IN}$

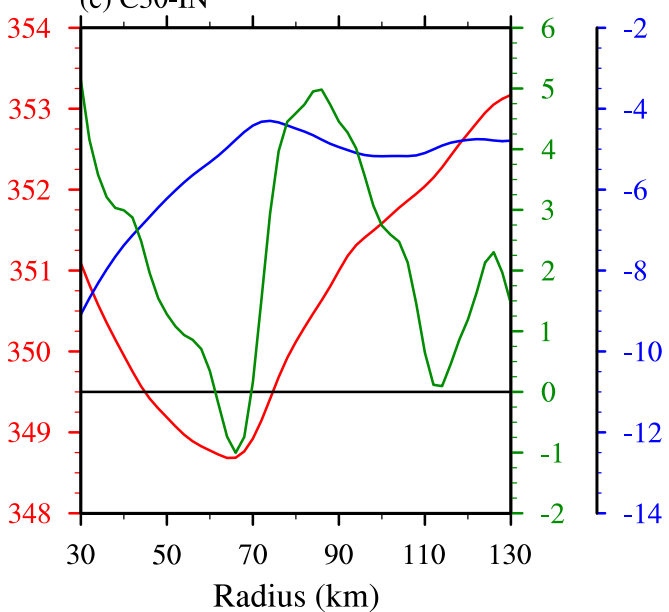

(b) $\mathrm{C} 40$

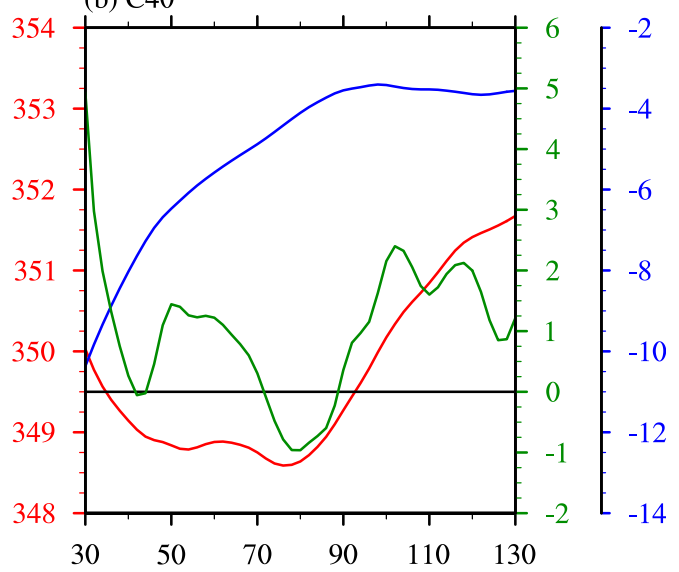

(d) C30-OUT

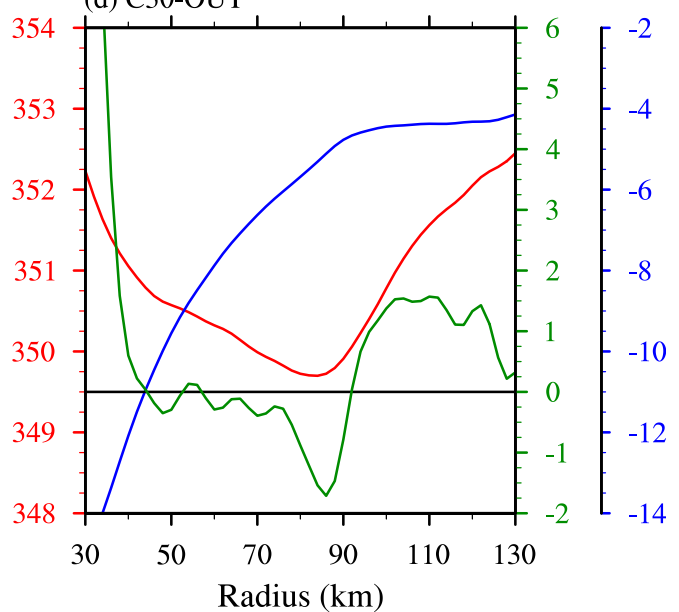

- Ept - Wa - Vr

FIG. 14. Radial profiles of azimuthal-mean equivalent potential temperature (red, K), vertical velocity (green, $\times 10^{-2} \mathrm{~m} \mathrm{~s}^{-1}$ ), and radial velocity (blue, $\mathrm{m} \mathrm{s}^{-1}$ ) at $1-\mathrm{km}$ height averaged from 70 to $90 \mathrm{~h}$ in (a) C20, (b) $\mathrm{C} 40$, (c) C30-IN, and (d) C30-OUT. The horizontal black lines represent zero vertical velocity for reference.

MSLP and a drop of MTW corresponding to one pronounced ERC event (Fig. 2). As shown in Fig. 13d, the outer convective rainbands are active outside a radius of $90 \mathrm{~km}$ before the ERC, similar to the case in C40. The contraction commences around $150 \mathrm{~h}$ and the robust SEF occurs at around $168 \mathrm{~h}$, both being later than those in C30. Subsequently, the primary eyewall dissipates and eventually is replaced by the contracted concentric convective ring at the end of this simulation.

It is instructive to illustrate the radial profiles of characteristic variables during the early stage that can present some hints of subsequent structural evolution. It is clear that the inner-core $\theta_{e}$ in $\mathrm{C} 20$ has the largest value among the sensitivity experiments (Figs. 14). Hence, the relatively warm and moist air surrounding the primary eyewall is less hostile to the TC intensity with a strong radial inflow in $\mathrm{C} 20$. In contrast to the maximum downward motion near a radius of $70 \mathrm{~km}$ in C30 and $80 \mathrm{~km}$ in $\mathrm{C} 40$, the azimuthal-mean descending motion is almost absent in the inner-core region in $\mathrm{C} 20$, such that no apparent moat appears (Figs. 13a and 14a). By comparison, the subsidence in C40 appears between 70and $90-\mathrm{km}$ radii, while the ascent exists outside $90 \mathrm{~km}$ (Fig. 14b). The minimum $\theta_{e}$ in C30-IN is located at a radius of $65 \mathrm{~km}$ while it is at $85 \mathrm{~km}$ in C30-OUT (Figs. 14c,d). Because the low-entropy air in C30-IN occurring at a small radius can be easily imported to the eyewall region, the storm has a quick decay with a weak radial inflow at a small radius. The peak outer upward motion in C30-IN has the largest magnitude and is located at the smallest radius among the sensitivity experiments, advancing the outward expansion of TC 

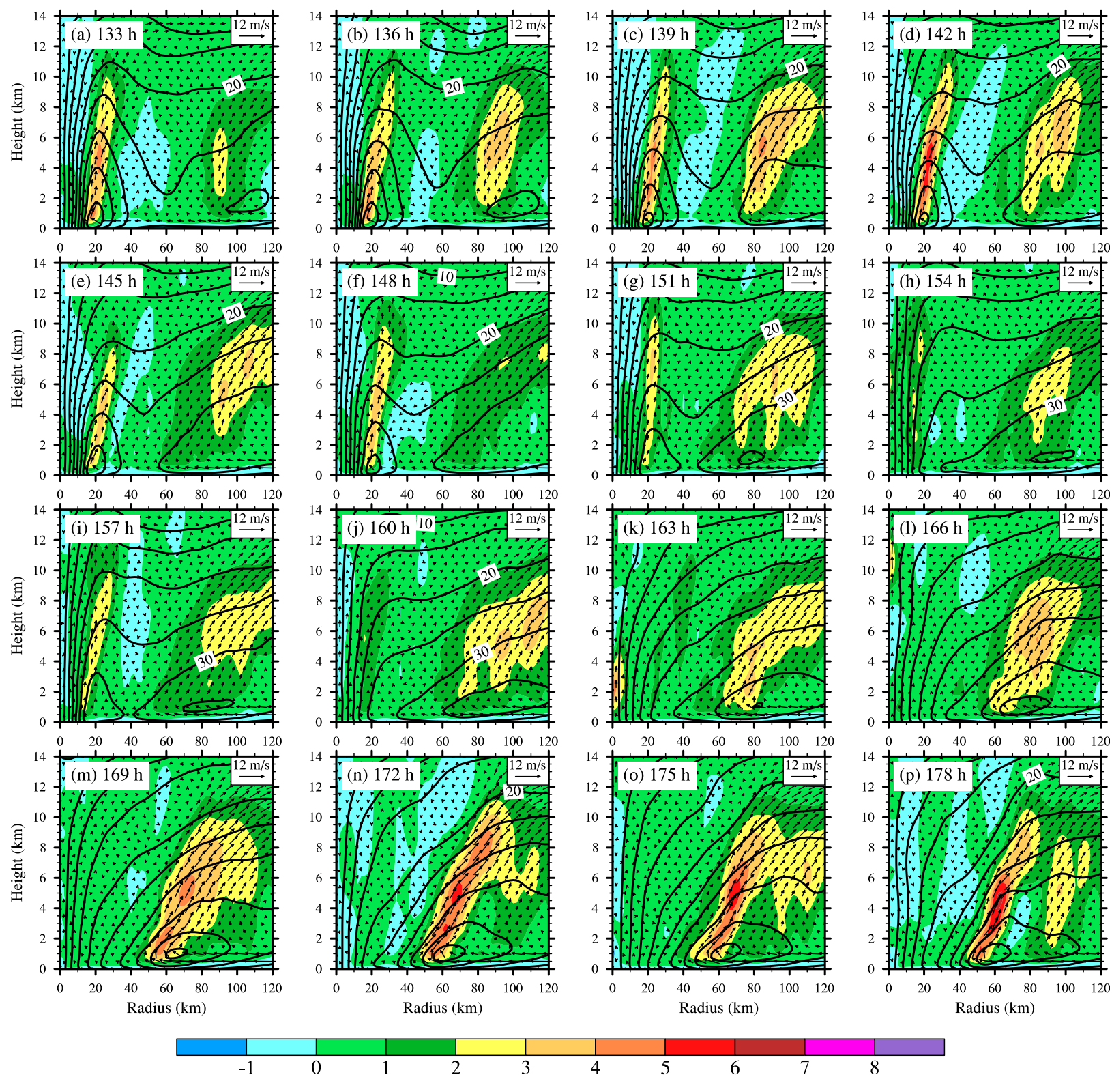

FIG. 15. As in Fig. 7, but in C40.

inner-core size and the establishment of moist convective updrafts outside the eyewall during the early period (Fig. 13c). The outward shift of increased cooling in C30OUT results in a relatively higher $\theta_{e}$ in the inner-core region than the counterparts in $\mathrm{C} 30, \mathrm{C} 40$, and $\mathrm{C} 30-\mathrm{IN}$. Because of less detriment to the primary eyewall convection, a comparatively strong radial inflow is maintained immediately outside the primary eyewall, and the storm still sustains a slow intensification in C30-OUT. The descending motion is located between 70- and 95-km radii, while the outer-core ascent exists outside $95 \mathrm{~km}$ (Fig. 14d).

\section{b. The unique ERCs in C40 and C30-OUT}

By comparing the structural evolution among C30, C40, and C30-OUT (Figs. 3b and 13b,d), two intriguing issues remain to be addressed. One is why the outer convective ring in $\mathrm{C} 40$ only experiences a slow shrinkage, and moreover just contracts to a radius of $60 \mathrm{~km}$ forming an AH-like structure. The other is why the time of SEF and ERC in C30-OUT is later than that in C30, but the secondary eyewall in C30-OUT has a more rapid inward contraction. The deep insights into these two issues would help us to understand 
what is responsible for the different behaviors of SEF and ERC.

To answer the first question, the radius-height cross sections of the azimuthal-mean storm structure in C40 are displayed in Fig. 15. The striking feature is that, in conjunction with the secondary diabatic heating centered at a radius of $90 \mathrm{~km}$, the secondary tangential wind maximum emerges at about $110 \mathrm{~km}$ at $133 \mathrm{~h} \mathrm{(Fig.} \mathrm{15a).}$ As the outer diabatic heating and inward momentum advected by the boundary layer inflows are strengthened, the outer secondary tangential winds become strong and penetrate radially inward as a wedge-like pattern (Figs. 15b-e), quite different from that in the first ERC of C30 in which the strong tangential wind expands outward from the primary eyewall region and then forms a secondary maximum in the SEF region. After a transient weakening of outer convection, the outer convective updrafts become vigorous again accompanied by the more pronounced radially inward penetration of the large secondary tangential wind within the boundary layer and the suppression of the primary eyewall (Figs. 15f-i). Later on, both the original primary eyewall convection and the corresponding maximum tangential wind dissipate, while the outer convective eyewall is progressively organized and moreover tilts outward with height (Figs. 15j-m). In particular, by $172 \mathrm{~h}$ (Fig. 15n) a robust outward-sloping convective eyewall is established, with a low-level maximum convective heating located at about $60 \mathrm{~km}$. Afterward, the vertically tilted outer eyewall nearly maintains at the same radius and the storm intensity keeps steady (Figs. 15n-p and 2). As discussed in the second ERC of $\mathrm{C} 30$, the large vertically outward slope of the outer convective eyewall can increase the low-level tangential wind outside the RMW, thus prohibiting the inward contraction of the outer eyewall and maintaining a large-eyed storm (Wang 2008b).

It is evident in Fig. 15 that the largely outward slope of the outer eyewall in $\mathrm{C} 40$ is characterized by the strong radial outflow and weak vertical motion above the boundary layer. Given that the slantwise updrafts can be viewed as the vector addition of vertical and radial velocities, the ratio of vertical velocity to radial velocity (hereafter referred to as tilt ratio) can be defined to quantitatively measure the extent of eyewall outward tilt (Stern and Nolan 2009). As clearly seen in Fig. 16, there are the strong and radially extending low-level radial outflows during the ERC in C40 and the second ERC in $\mathrm{C} 30$, whereas both the magnitude and radial extent of radial outflow are small during the first ERC in C30 and C30-OUT (Figs. 16a-c), which contributes to the discrepancy in tilt ratio (Figs. 16d-f). Furthermore, the difference in the radial outflow mainly arises from the distinct distribution of deep-layer AF calculated in Eq. (3). During the ERC in C40 and the second ERC in $\mathrm{C} 30$, there are massive outer tangential wind maximum at the low levels (Figs. 15l-n and 7n-p). As a result, the positive AF averaged over the heights of $1.5-5 \mathrm{~km}$ is more robust and radially extensive during the ERC in $\mathrm{C} 40$ and the second ERC in $\mathrm{C} 30$, causing the stronger radially outward-pointed supergradient winds. By comparison, the deep-layer AF during the first ERC of C30 is small (Fig. 16b) and the positive AF is only confined near the top of boundary layer as implied in Fig. 11a, quite different from the large deep-tropospheric positive AF during the secondary ERC of C30.

The vertically extending and strengthened positive AF during the ERC in C40 is intimately related to the structural evolution of secondary tangential flow outside the primary eyewall. As shown in Fig. 15, detached from the maximum tangential wind in the primary eyewall, the secondary tangential wind associated with the outer convective heating is accelerated prior to the ERC, which results from the enhanced radial advection of absolute vorticity $\left(\bar{u} \overline{\varsigma_{a}}\right)$ in the outer-core region because of the strengthening of the outer convective rainbands (not shown). In collaboration with the localized weak pressure radial gradient $(-\partial \bar{p} / \bar{\rho} \partial r)$, the large bulk of strong outer tangential flow in C40 generates the deep-tropospheric outward-directed AF and thus radial outflows based on Eq. (3) (Fig. 16b). In addition, the moderate vertical velocity in the ERC of C40 can assure the coherently small tilt ratio in the outer eyewall, as is the case in the second ERC of C30. In contrast, because of the vigorous updrafts and small radial outflows, the primary eyewalls in the sensitivity experiments and the secondary eyewalls in the first ERC of $\mathrm{C} 30$ and $\mathrm{C} 30-\mathrm{OUT}$ exhibit a relatively radially narrow and vertically aligned structure, with the large tilt ratio (Figs. 16d-f).

Regarding the contraction speed of the outer concentric eyewall during the ERCs in C40 and C30-OUT, it is clearly depicted in Figs. 13b,d that the outer convective eyewall has a slow contraction in $\mathrm{C} 40$, while it has a rapid contraction to the radius of the original primary eyewall in C430-OUT. Owing to the quick collapse of the primary eyewall, $\mathrm{C} 40$ possesses the weak intensity and low-level inflows inside a radius of about $80 \mathrm{~km}$ before and during the ERC. Accordingly, the outer convective eyewall in $\mathrm{C} 40$ has a slow inward displacement caused by the attenuation of the inward transport of large absolute angular momentum $(M)$ outside the primary eyewall. On the contrary, the rapid contraction of the outer concentric eyewall in C30-OUT can be attributed to the strong boundary layer inflows as displayed in Fig. 14d, which advect the large $M$ surface 
(a) C40: AF and $\mathrm{U}$

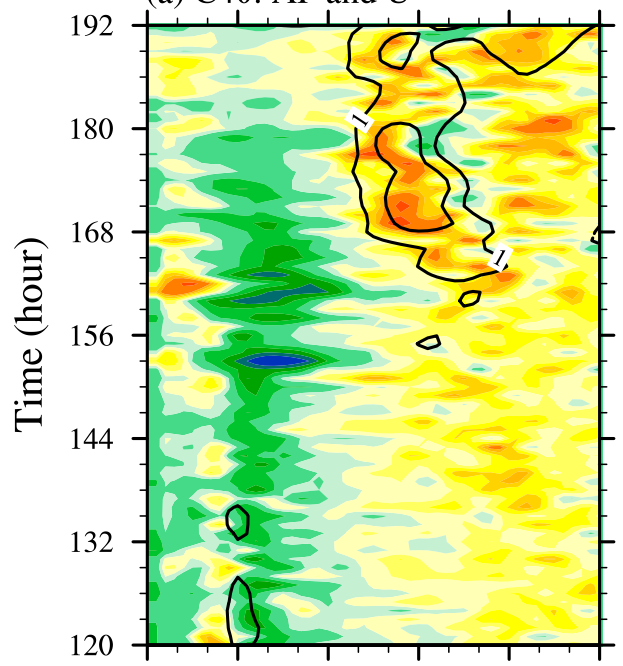

(b) C30: $\mathrm{AF}$ and $\mathrm{U}$

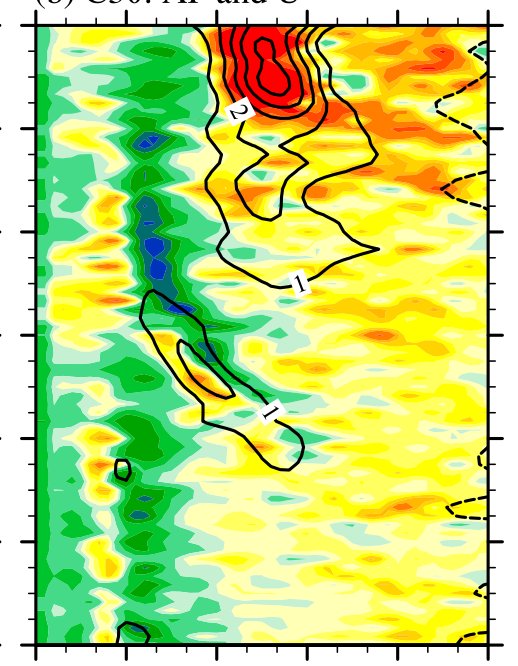

(c) C30-OUT: AF and U

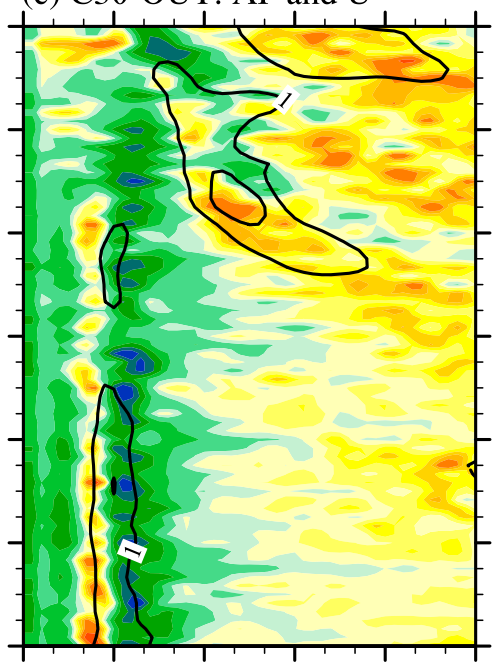

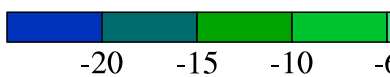
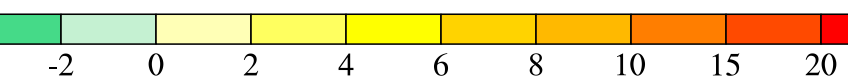

(d) $\mathrm{C} 40: \mathrm{W} / \mathrm{U}$ and $\mathrm{W}$

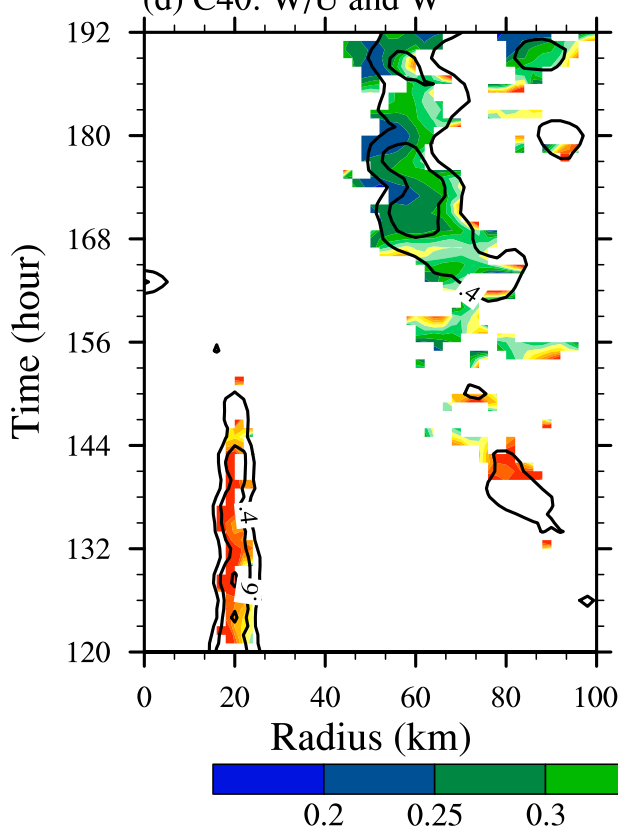

(e) $\mathrm{C} 30: \mathrm{W} / \mathrm{U}$ and $\mathrm{W}$

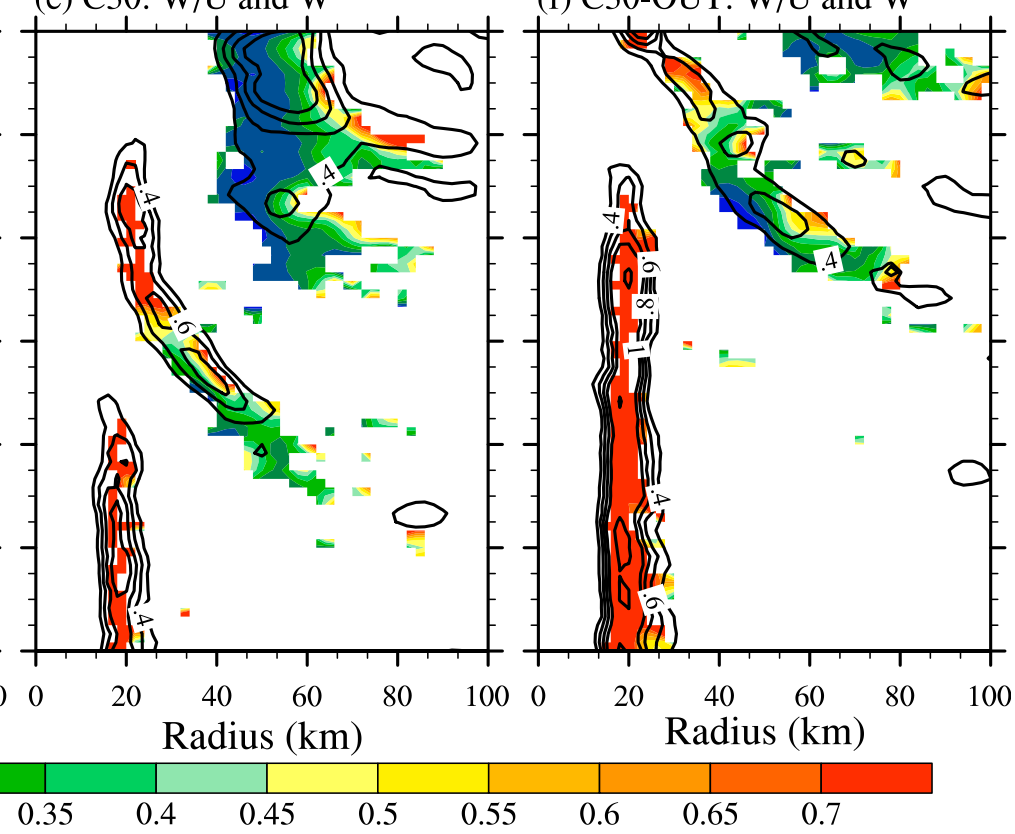

FIG. 16. Radius-time Hovmöller diagrams of azimuthal-mean (a)-(c) agradient force (color shaded, $\mathrm{m} \mathrm{s}^{-1} \mathrm{~h}^{-1}$ ) and radial velocity (contours, $\mathrm{m} \mathrm{s}^{-1}$ ) and (d)-(f) ratio of vertical velocity to radial velocity (color shaded) and vertical velocity (contours, $\mathrm{m} \mathrm{s}^{-1}$ ) averaged over heights from 1.5 to $5 \mathrm{~km}$ in (a),(d) C40; (b),(e) C30; and (c),(f) C30-OUT.

(or eyewall) more radially inward to replace the collapsed inner eyewall.

In C30-OUT, another striking difference is that the ERC occurs later than that in C30 by about $24 \mathrm{~h}$. Figure 17 displays that the outer diabatic heating related to the ERC originates around a radius of $100 \mathrm{~km}$ (Figs. 17a-d), because of the outward displacement of increased low-level cooling. Starting from about $158 \mathrm{~h}$, the outer convective band commences to intensify and concurrently contract inward quickly, with the boundary layer part of the eyewall arriving at a radius of $40 \mathrm{~km}$ around $173 \mathrm{~h}$ (Figs. 17e-g). Afterward, the secondary convective ring becomes more upright and radially narrow, and then contracts to the radius of $20 \mathrm{~km}$ completing an ERC at $191 \mathrm{~h}$ (Figs. 17k-p). Meanwhile, one new outward-tilted convective heating develops outside 

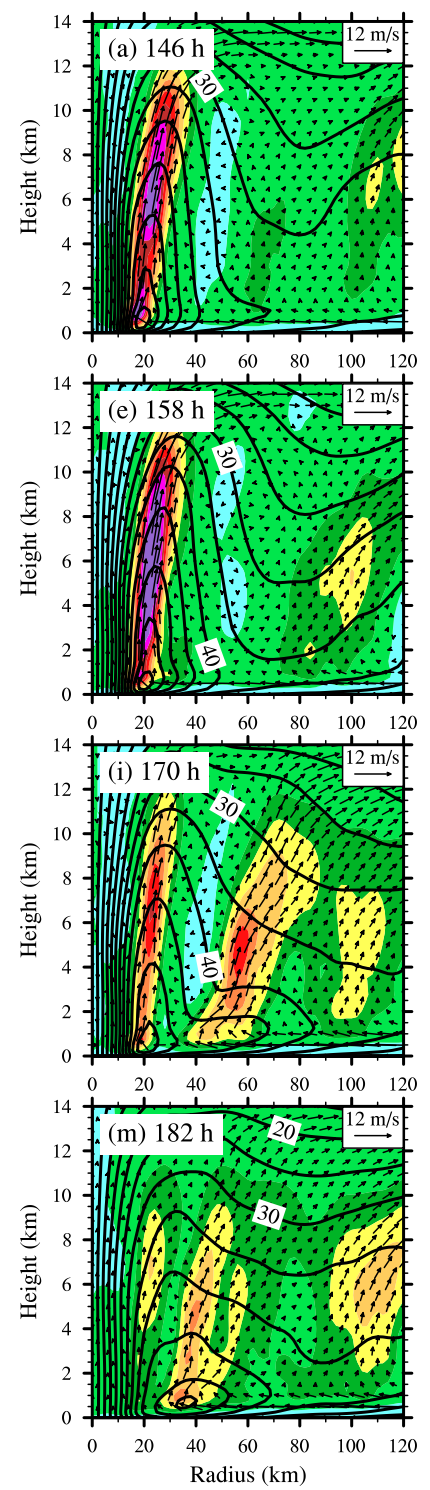
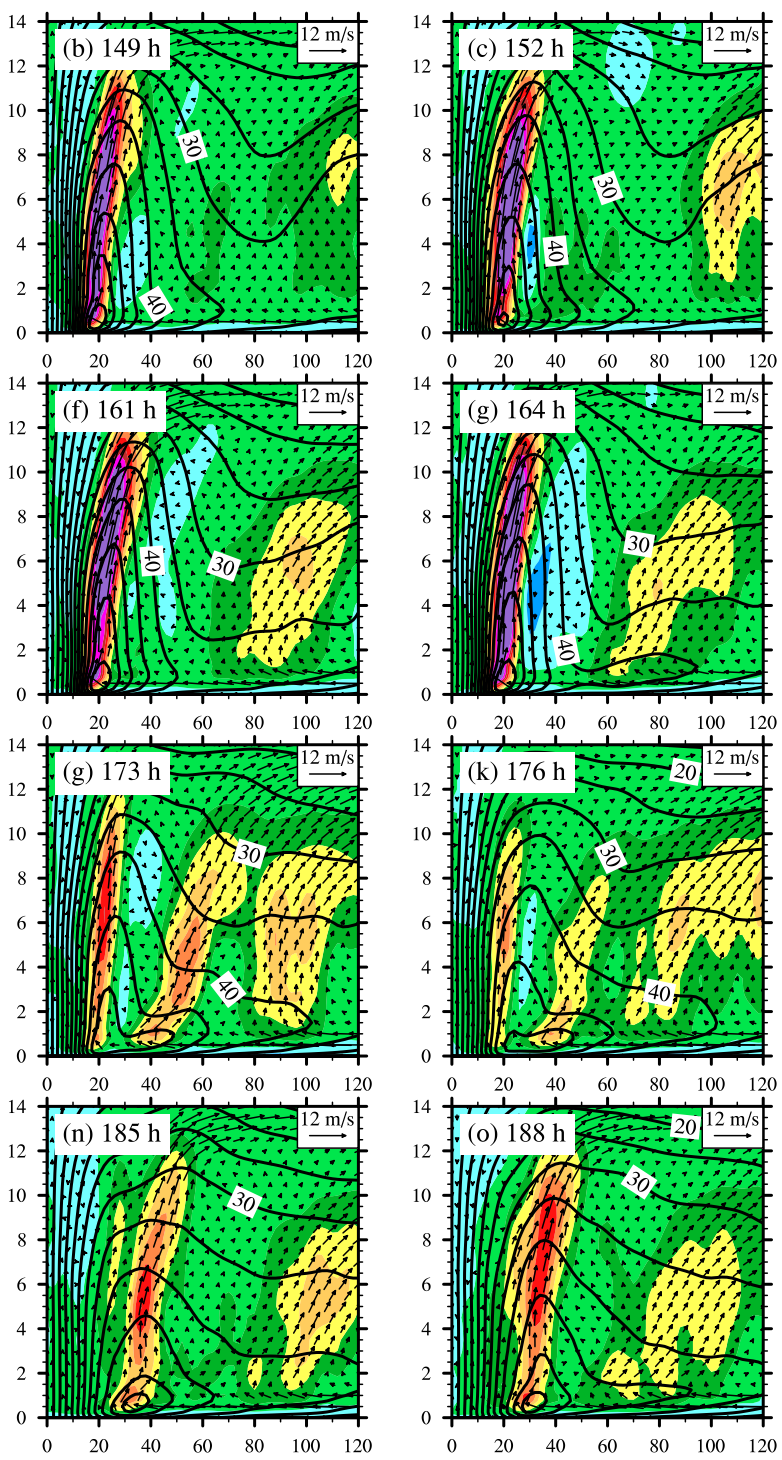

Radius $(\mathrm{km})$
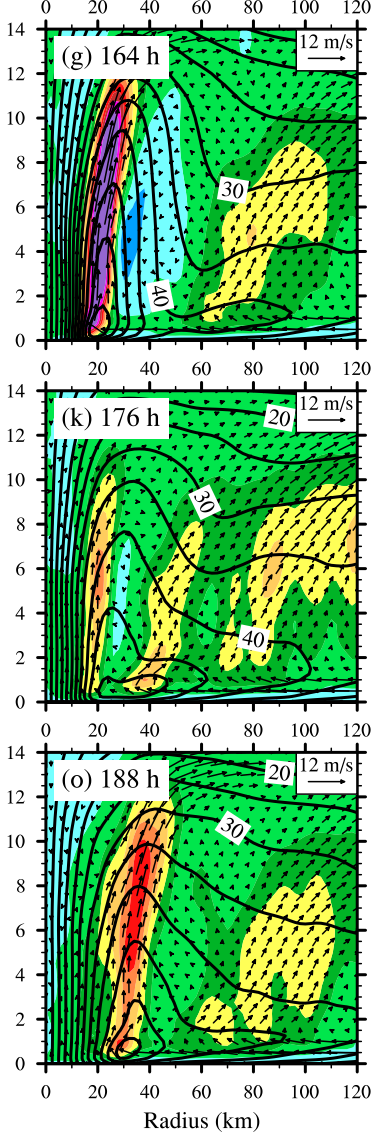

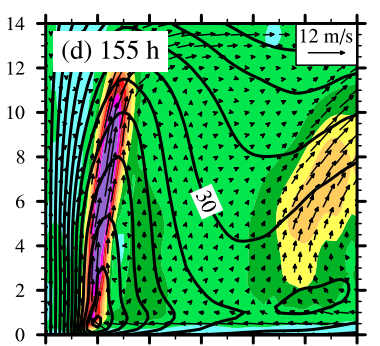

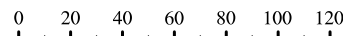
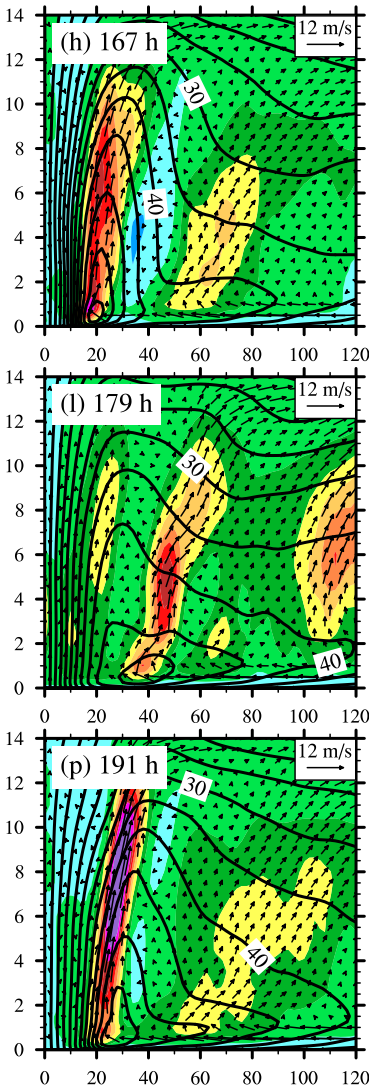

Radius (km)

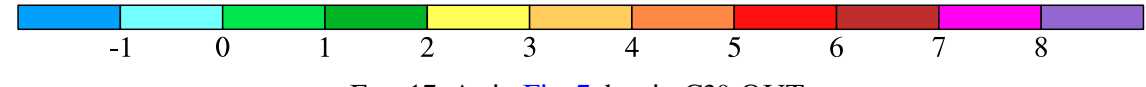

FIG. 17. As in Fig. 7, but in C30-OUT.

the inner eyewall, ultimately also forming a large-eyed storm as the case in C30. By comparison, the active outer convective updrafts prior to the first SEF in C30 mainly occurs between radii of 80 and $90 \mathrm{~km}$, while those in C30-OUT are located outside $90 \mathrm{~km}$ (cf. Figs. $3 b$ and $13 d$; Figs. 5 b and $14 d$ ). As a result, the lowlevel inertial stability is higher in the outer rainband region in C30 than in C30-OUT (Fig. 18). As discussed previously, the large inertial stability can effectively warm the atmospheric column, lower the surface pressure, and strengthen the near-surface wind, which in turn further enhances the low-level convergence and surface entropy flux, thus favoring the convective burst. Consequently, this positive feedback leads to an earlier onset of ERC in C30 than in C30-OUT.

\section{Conclusions and discussion}

In this study, the effect of increased low-level innercore diabatic cooling on SEF and ERC is examined using idealized WRF simulations on an $f$ plane. In CTL initialized with an axisymmetric vortex, the storm experiences a rapid intensification accompanied by a slow outward expansion of RMW. In addition, the storm 
(a) $\mathrm{C} 30$

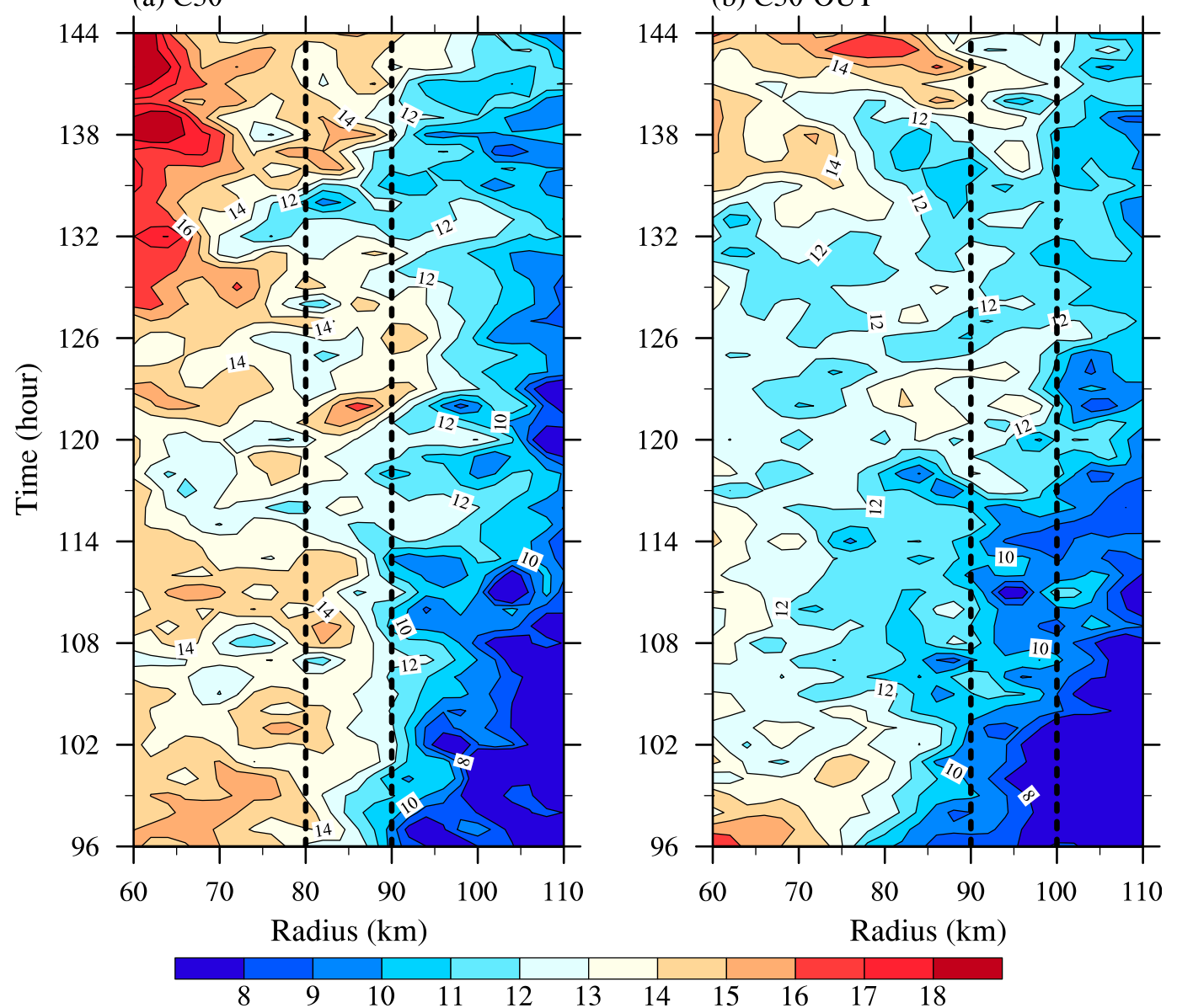

FIG. 18. Radius-time Hovmöller diagrams of azimuthal-mean normalized inertial stability averaged over heights from 1 to $5 \mathrm{~km}$ in (a) C30 and (b) C30-OUT. The active outer rainbands in C30 and C30-OUT mainly fall in the regions between the two black vertical dashed lines.

lacks active outer rainbands, eventually forming a compact inner-core structure. The primary sensitivity experiment $(\mathrm{C} 30)$ is initialized at $62 \mathrm{~h}$ of CTL but with the low-level diabatic cooling increased by $30 \%$ within the annulus of $40-80 \mathrm{~km}$. The distinction shows that the SEF in C30 emerges around $148 \mathrm{~h}$, and then contracts inward to replace the dissipated inner eyewall, completing the first ERC. Later, a thick outer eyewall with a largely outward slope develops in the inner-core region during the second ERC, finally evolving into a largesized storm.

Corresponding to the increased low-level inner-core cooling in $\mathrm{C} 30$, the descending motion and divergence are present within the inner-core boundary layer, while the convective rainbands in the outer-core region become active because of the boundary layer convergence related to the decelerated inflow or even outflow. The outward expansion of the strong wind field induced by the active outer rainbands not only increases the inner-core surface heat flux in collaboration with the large air-sea thermal difference but also generates the ambient dynamic parameters favorable for SEF, such as effective $\beta$ skirt, large filamentation time, and high inertial stability. In addition, the broadening of large tangential wind contributes to two boundary layer processes facilitating SEF, that is, unbalanced dynamics related to agradient flow and frictionally forced updraft related to vorticity. As a result, a deep convective burst is triggered at a radius of $50 \mathrm{~km}$, leading to a well-defined SEF at about $148 \mathrm{~h}$ and then completing the first ERC. During the secondary ERC, the deep-tropospheric outward-directed supergradient flows caused by the strong tangential winds enhance the radial outflows above the boundary layer, producing a largely outwardtilted outer eyewall and thus inhibiting its contraction.

To further examine the sensitivity of TC structure and intensity change to the strength and radial location of low-level cooling, the extra four sensitivity experiments 
are supplemented, in which the diabatic cooling is increased by $20 \%$ and $40 \%$, and shifted radially inward and outward by $10 \mathrm{~km}$ in C20, C40, C30-IN, and C30-OUT, respectively. The results show that, in C20 and C30-IN, there are not conspicuous downdrafts in the inner-core region, and moreover, the outer convective rainbands develop close to the primary eyewall. As a result, neither $\mathrm{C} 20$ nor C30-IN exhibits a pronounced moat and SEF. In contrast, the moat is evident in C40 and C30-OUT with the outer convective updrafts initiated at a large radius. The unique feature in C40 takes on a slow contraction of the outer eyewall and eventually an AH-like storm without multiple ERCs as in C30. During the ERC in C40, a separated and massive tangential wind maximum occurs along with the outer convective heating, which generates the large deeptropospheric outward-directed AF and thus radial outflow above the boundary layer. With the aid of the weak inner-core boundary layer inflow, the largely outwardtilted eyewall shrinks slowly, preventing it from contracting more radially inward. As for C30-OUT, the distinctive behavior is that the SEF is later than that in C30, but with an expeditious ERC. As opposed to the case in $\mathrm{C} 30$, the outer convective rainbands related to the SEF in C30-OUT occur at a larger radius with a relatively lower inertial stability, hence postponing the SEF. However, the strong boundary layer inflow can rapidly advect the concentric convective ring to a small radius, forming a more upright eyewall at the end of simulation in C30-OUT.

In addition, this study also provides some implications. Even if outer convective rainbands are not triggered by external or other internal forcings, persistent inner-core cooling is likely to dynamically and thermodynamically initiate active outer convection that is crucial for SEF. The argument in this study is also implied by Wang (2002), who showed in his full-physics model simulations that when the melting of snow and graupel and the evaporation of rain are turned off, characterized by the decrease in inner-core diabatic cooling, the outer spiral rainbands are largely suppressed or even disappear. Regarding the role of microphysics processes in SEF and ERC, Zhu and Zhu (2015) pointed out that the large snow terminal velocity and its induced cooling below the freezing level can cause strong downdrafts that penetrate underneath the peripheral updrafts and promote strong convective eyewall. As a response, the moist air with a large entropy is transported inward by the strong radial inflow to promote shallow convection in the moat. As a result, the outer concentric eyewall moves too close to the inner eyewall to identify a welldefined secondary eyewall. In contrast, the small snow terminal velocity causes weak evaporative cooling and results in the weak eyewall updrafts, which is unable to generate a radial inflow sufficiently strong to foster shallow convection in the moat, and thus allows a clean moat between the inner and outer eyewalls. Besides, Zhou et al. (2011) found that the enhanced concentration of ice particles at the upper-tropospheric outflow can produce a noticeable subsidence region (moat) surrounding the primary eyewall, and moreover, the secondary eyewall forms at an increased radius. In this study, the examination of the sensitivity of SEF and ERC to cooling in a specific region can help isolate the effect of diabatic process in the region of interest on structural evolution and intensity change. Based on the artificially modified diabatic cooling, the results in this study also hint that the specification of key parameters employed in model cumulus and microphysics schemes may to a large extent affect TC structural evolution and intensity change. Especially for the representation of stratiform-type precipitation featured by a salient lowlevel cooling, the overestimation of inner-core diabatic cooling and related downdrafts would tend to enhance outer spiral rainbands and thus increase the possibility of SEF. Therefore, the cumulus and microphysics schemes and their coupling to interior flows need to be adequately represented in numerical models to improve the prediction of SEF timing and preferred location. For real TCs, future work should be devoted to exploring how large-scale environmental conditions govern dynamic and thermodynamic behaviors in the TC innercore region that would greatly affect TC structure and intensity change.

Acknowledgments. I acknowledge the valuable comments of three anonymous reviewers. I am very grateful to Prof. Chun-Chieh Wu, Dr. Yi-Hsuan Huang, and Dr. Xiaodong Tang for their constructive discussions. This study is supported by the National Natural Science Foundation of China (Grants 41775063 and 41475074), the National Basic Research Program of China (Grant 2014CB953902), and the open project of Key Laboratory of Meteorological Disaster of Ministry of Education, NUIST (KLME1604).

\section{REFERENCES}

Bender, M. A., 1997: The effect of relative flow on the asymmetric structure in the interior of hurricanes. J. Atmos. Sci., 54, 703-724, https://doi.org/10.1175/1520-0469(1997)054<0703: TEORFO $>2.0 . \mathrm{CO} ; 2$.

Bui, H. H., R. K. Smith, M. T. Montgomery, and J. Peng, 2009: Balanced and unbalanced aspects of tropical cyclone intensification. Quart. J. Roy. Meteor. Soc., 135, 1715-1731, https://doi.org/ 10.1002/qj.502.

Chen, G., 2016: Determination of the effect of initial inner-core structure on tropical cyclone intensification and track on a 
beta plane. Adv. Atmos. Sci., 33, 945-954, https://doi.org/ 10.1007/s00376-016-5241-9.

— C.-C. Wu, and Y.-H. Huang, 2018: The role of near-core convective and stratiform heating/cooling in tropical cyclone structure and intensity. J. Atmos. Sci., 75, 297-326, https:// doi.org/10.1175/JAS-D-17-0122.1.

Dai, Y., S. J. Majumdar, and D. S. Nolan, 2017: Secondary eyewall formation in tropical cyclones by outflow-jet interaction. J. Atmos. Sci., 74, 1941-1958, https://doi.org/10.1175/JASD-16-0322.1.

Didlake, A. C., Jr., and R. A. Houze Jr., 2009: Convective-scale downdrafts in the principal rainband of Hurricane Katrina (2005). Mon. Wea. Rev., 137, 3269-3293, https://doi.org/ 10.1175/2009MWR2827.1.

Dudhia, J., 1989: Numerical study of convection observed during the Winter Monsoon Experiment using a mesoscale two-dimensional model. J. Atmos. Sci., 46, 3077-3107, https:// doi.org/10.1175/1520-0469(1989)046<3077:NSOCOD>2.0.CO;2.

Ebita, A., and Coauthors, 2011: The Japanese 55-year Reanalysis "JRA-55": An interim report. SOLA, 7, 149-152, https:// doi.org/10.2151/sola.2011-038.

Emanuel, K. A., 1986: An air-sea interaction theory for tropical cyclones. Part I: Steady-state maintenance. J. Atmos. Sci., 43, 585-605, https://doi.org/10.1175/1520-0469(1986)043<0585: AASITF $>2.0 . \mathrm{CO} ; 2$.

Fang, J., and F. Zhang, 2012: Effect of beta shear on simulated tropical cyclones. Mon. Wea. Rev., 140, 3327-3346, https://doi.org/ 10.1175/MWR-D-10-05021.1.

Fudeyasu, H., and Y. Wang, 2011: Balanced contribution to the intensification of a tropical cyclone simulated in TCM4: Outer-core spinup process. J. Atmos. Sci., 68, 430-449, https:// doi.org/10.1175/2010JAS3523.1.

Guinn, T. A., and W. H. Schubert, 1993: Hurricane spiral bands. J. Atmos. Sci., 50, 3380-3403, https://doi.org/10.1175/15200469(1993) $050<3380: \mathrm{HSB}>2.0 . \mathrm{CO} ; 2$.

Hawkins, H., 1983: Hurricane Allen and island obstacles. J. Atmos. Sci., 40, 1360-1361, https://doi.org/10.1175/1520-0469(1983) 040<1360:HAAIO $>2.0$. CO; 2 .

Hence, D. A., and R. A. Houze Jr., 2008: Kinematic structure of convective-scale elements in the rainbands of Hurricanes Katrina and Rita (2005). J. Geophys. Res., 113, D15108, https://doi.org/10.1029/2007JD009429.

Hong, S.-Y., J. Dudhia, and S.-H. Chen, 2004: A revised approach to ice microphysical processes for the bulk parameterization of clouds and precipitation. Mon. Wea. Rev., 132, 103-120, https:// doi.org/10.1175/1520-0493(2004)132<0103:ARATIM>2.0.CO;2.

Houze, R. A., Jr., S. S. Chen, B. F. Smull, W.-C. Lee, and M. M. Bell, 2007: Hurricane intensity and eyewall replacement. Science, 315, 1235-1239, https://doi.org/10.1126/science.1135650.

Huang, Y.-H., M. T. Montgomery, and C.-C. Wu, 2012: Concentric eyewall formation in Typhoon Sinlaku (2008). Part II: Axisymmetric dynamical processes. J. Atmos. Sci., 69, 662-674, https://doi.org/10.1175/JAS-D-11-0114.1.

Kepert, J. D., 2013: How does the boundary layer contribute to eyewall replacement cycles in axisymmetric tropical cyclones? J. Atmos. Sci., 70, 2808-2830, https://doi.org/10.1175/JAS-D13-046.1.

Knaff, J. A., J. P. Kossin, and M. DeMaria, 2003: Annular hurricanes. Wea. Forecasting, 18, 204-223, https://doi.org/10.1175/ 1520-0434(2003)018<0204:AH>2.0.CO;2.

Kossin, J. P., and M. Sitkowski, 2009: An objective model for identifying secondary eyewall formation in hurricanes. Mon. Wea. Rev., 137, 876-892, https://doi.org/10.1175/2008MWR2701.1.
Kuo, H.-C., L.-Y. Lin, C.-P. Chang, and R. T. Williams, 2004: The formation of concentric vorticity structures in typhoons. J. Atmos. Sci., 61, 2722-2734, https://doi.org/10.1175/JAS3286.1.

— W. W. Schubert, C.-L. Tsai, and Y.-F. Kuo, 2008: Vortex interactions and barotropic aspects of concentric eyewall formation. Mon. Wea. Rev., 136, 5183-5198, https://doi.org/ 10.1175/2008MWR2378.1.

_- C.-P. Chang, Y.-T. Yang, and H.-J. Jiang, 2009: Western North Pacific typhoons with concentric eyewalls. Mon. Wea. Rev., 137, 3758-3770, https://doi.org/10.1175/2009MWR2850.1.

Li, Q., Y. Wang, and Y. Duan, 2014: Effects of diabatic heating and cooling in the rapid filamentation zone on structure and intensity of a simulated tropical cyclone. J. Atmos. Sci., 71, 31443163, https://doi.org/10.1175/JAS-D-13-0312.1.

Mlawer, E. J., S. J. Taubman, P. D. Brown, M. J. Iacono, and S. A. Clough, 1997: Radiative transfer for inhomogeneous atmospheres: RRTM, a validated correlated-k model for the longwave. J. Geophys. Res., 102, 16 663-16682, https://doi.org/ 10.1029/97JD00237.

Molinari, J., and D. Vollaro, 1990: External influences on hurricane intensity. Part II: Vertical structure and response of the hurricane vortex. J. Atmos. Sci., 47, 1902-1918, https://doi.org/ 10.1175/1520-0469(1990)047<1902:EIOHIP>2.0.CO;2.

Montgomery, M. T., and R. J. Kallenbach, 1997: A theory for vortex Rossby-waves and its application to spiral bands and intensity changes in hurricanes. Quart. J. Roy. Meteor. Soc., 123, 435-465, https://doi.org/10.1002/qj.49712353810.

Moon, Y., and D. S. Nolan, 2010: The dynamic response of the hurricane wind field to spiral rainband heating. J. Atmos. Sci., 67, 1779-1805, https://doi.org/10.1175/2010JAS3171.1.

Noh, Y., W. Cheon, S. Hong, and S. Raasch, 2003: Improvement of the K-profile model for the planetary boundary layer based on large eddy simulation data. Bound.-Layer Meteor., 107, 401427, https://doi.org/10.1023/A:1022146015946.

Nong, S., and K. Emanuel, 2003: A numerical study of the genesis of concentric eyewalls in hurricanes. Quart. J. Roy. Meteor. Soc., 129, 3323-3338, https://doi.org/10.1256/qj.01.132.

Powell, M. D., 1990: Boundary layer structure and dynamics in outer hurricane rainbands. Part II: Downdraft modification and mixed layer recovery. Mon. Wea. Rev., 118, 918-938, https:// doi.org/10.1175/1520-0493(1990)118<0918:BLSADI >2.0.CO;2.

Qiu, X., and Z. M. Tan, 2013: The roles of asymmetric inflow forcing induced by outer rainbands in tropical cyclone secondary eyewall formation. J. Atmos. Sci., 70, 953-974, https:// doi.org/10.1175/JAS-D-12-084.1.

—_— - and Q. Xiao, 2010: The roles of vortex Rossby waves in hurricane secondary eyewall formation. Mon. Wea. Rev., $\mathbf{1 3 8}$ 2092-2109, https://doi.org/10.1175/2010MWR3161.1.

Rogers, R., 2010: Convective-scale structure and evolution during a high-resolution simulation of tropical cyclone rapid intensification. J. Atmos. Sci., 67, 44-70, https://doi.org/10.1175/ 2009JAS3122.1.

Rotunno, R., and K. A. Emanuel, 1987: An air-sea interaction theory for tropical cyclones. Part II: Evolutionary study using a nonhydrostatic axisymmetric numerical model. J. Atmos. Sci., 44, 542-561, https://doi.org/10.1175/1520-0469 (1987)044<0542:AAITFT>2.0.CO;2.

Rozoff, C. M., W. H. Schubert, B. D. McNoldy, and J. P. Kossin, 2006: Rapid filamentation zones in intense tropical cyclones. J. Atmos. Sci., 63, 325-340, https://doi.org/10.1175/JAS3595.1.

,-- , and J. P. Kossin, 2008: Some dynamical aspects of tropical cyclone concentric eyewalls. Quart. J. Roy. Meteor. Soc., 134, 583-593, https://doi.org/10.1002/qj.237. 
D. S. Nolan, J. P. Kossin, F. Zhang, and J. Fang, 2012: The roles of an expanding wind field and inertial stability in tropical cyclone secondary eyewall formation. J. Atmos. Sci., 69 , 2621-2643, https://doi.org/10.1175/JAS-D-11-0326.1.

Sawada, M., and T. Iwasaki, 2010: Impacts of evaporation from raindrops on tropical cyclones. Part II: Features of rainbands and asymmetric structure. J. Atmos. Sci., 67, 84-96, https:// doi.org/10.1175/2009JAS3195.1.

Schubert, W. H., and J. J. Hack, 1982: Inertial stability and tropical cyclone development. J. Atmos. Sci., 39, 1687-1697, https:// doi.org/10.1175/1520-0469(1982)039<1687:ISATCD>2.0.CO;2.

Shapiro, L. J., and H. E. Willoughby, 1982: The response of balanced hurricanes to local sources of heat and momentum. J. Atmos. Sci., 39, 378-394, https://doi.org/10.1175/1520-0469 (1982)039<0378:TROBHT>2.0.CO;2.

Sitkowski, M., J. P. Kossin, and C. M. Rozoff, 2011: Intensity and structure changes during hurricane eyewall replacement cycles. Mon. Wea. Rev., 139, 3829-3847, https://doi.org/10.1175/ MWR-D-11-00034.1.

Stern, D. P., and D. S. Nolan, 2009: Reexamining the vertical structure of tangential winds in tropical cyclones: Observations and theory. J. Atmos. Sci., 66, 3579-3600, https://doi.org/ 10.1175/2009JAS2916.1.

Terwey, W. D., and M. T. Montgomery, 2008: Secondary eyewall formation in two idealized, full-physics modeled hurricanes. J. Geophys. Res., 113, D12112, https://doi.org/10.1029/ 2007JD008897.

Wang, X., Y. Ma, and N. E. Davidson, 2013: Secondary eyewall formation and eyewall replacement cycles in a simulated hurricane: Effect of the net radial force in the hurricane boundary layer. J. Atmos. Sci., 70, 1317-1341, https://doi.org/ 10.1175/JAS-D-12-017.1.

Wang, Y., 2002: An explicit simulation of tropical cyclones with a triply nested movable mesh primitive equation model: TCM3. Part II: Model refinements and sensitivity to cloud microphysics parameterization. Mon. Wea. Rev., 130, 3022-3036, https://doi.org/10.1175/1520-0493(2002)130<3022:AESOTC $>2.0$. $\mathrm{CO} ; 2$. 2008a: Rapid filamentation zone in a numerically simulated tropical cyclone. J. Atmos. Sci., 65, 1158-1181, https://doi.org/ 10.1175/2007JAS2426.1.

, 2008b: Structure and formation of an annular hurricane simulated in a fully compressible, nonhydrostatic modelTCM4. J. Atmos. Sci., 65, 1505-1527, https://doi.org/10.1175/ 2007JAS2528.1.

_ 2009: How do outer spiral rainbands affect tropical cyclone structure and intensity? J. Atmos. Sci., 66, 1250-1273, https:// doi.org/10.1175/2008JAS2737.1.

Willoughby, H. E., J. A. Clos, and M. G. Shoreibah, 1982: Concentric eye walls, secondary wind maxima, and the evolution of the hurricane vortex. J. Atmos. Sci., 39, 395-411, https://doi.org/ 10.1175/1520-0469(1982)039<0395:CEWSWM >2.0.CO;2.

— , F. D. Marks Jr., and R. J. Feinberg, 1984: Stationary and moving convective bands in hurricanes. J. Atmos. Sci., 41, 3189-3211, https://doi.org/10.1175/1520-0469(1984)041<3189: SAMCBI $>2.0 . \mathrm{CO} ; 2$.

Wu, C.-C., Y.-H. Huang, and G.-Y. Lien, 2012: Concentric eyewall formation in Typhoon Sinlaku (2008). Part I: Assimilation of T-PARC data based on the ensemble Kalman filter (EnKF). Mon. Wea. Rev., 140, 506-527, https://doi.org/10.1175/MWRD-11-00057.1.

Zhou, X., and B. Wang, 2009: From concentric eyewall to annular hurricane: A numerical study with the cloud-resolved WRF model. Geophys. Res. Lett., 36, L03802, https://doi.org/ 10.1029/2008GL036854.

,,-- X. Ge, and T. Li, 2011: Impact of secondary eyewall heating on tropical cyclone intensity change. J. Atmos. Sci., 68 , 450-456, https://doi.org/10.1175/2010JAS3624.1.

Zhu, Z., and P. Zhu, 2014: The role of outer rainband convection in governing the eyewall replacement cycle in numerical simulations of tropical cyclones. J. Geophys. Res. Atmos., 119, 8049-8072, https://doi.org/10.1002/2014JD021899.

, and - 2015: Sensitivities of eyewall replacement cycle to model physics, vortex structure, and background winds in numerical simulations of tropical cyclones. J. Geophys. Res. Atmos., 120, 590-622, https://doi.org/10.1002/2014JD022056. 